\title{
Analysis of a Cylindrical Specimen Heated by an Impinging Hot Hydrogen Jet
}

\author{
Ten-See Wang ${ }^{*}$, Van Luong ${ }^{\dagger}$, John Foote ${ }^{\ddagger}$, and Ron Litchford ${ }^{\S}$ \\ NASA Marshall Space Flight Center, Huntsville, Alabama 35812 \\ and \\ Yen-Sen Chen ${ }^{* *}$ \\ Engineering Sciences, Inc., Huntsville, Alabama, 35815
}

\begin{abstract}
A computational conjugate heat transfer methodology was developed, as a first step towards an efficient and accurate multiphysics, thermo-fluid computational methodology to predict environments for hypothetical solid-core, nuclear thermal engine thrust chamber and components. A solid conduction heat transfer procedure was implemented onto a pressure-based, multidimensional, finite-volume, turbulent, chemically reacting, thermally radiating, and unstructured grid computational fluid dynamics formulation. The conjugate heat transfer of a cylindrical material specimen heated by an impinging hot hydrogen jet inside an enclosed test fixture was simulated and analyzed. The solid conduction heat transfer procedure was anchored with a standard solid heat transfer code. Transient analyses were then performed with variable thermal conductivities representing three composites of a material utilized as flow element in a legacy engine test. It was found that material thermal conductivity strongly influences the transient heat conduction characteristics. In addition, it was observed that high thermal gradient occur inside the cylindrical specimen during an impulsive or a $10 \mathrm{~s}$ ramp start sequence, but not during steady-state operations.
\end{abstract}

\section{Nomenclature}

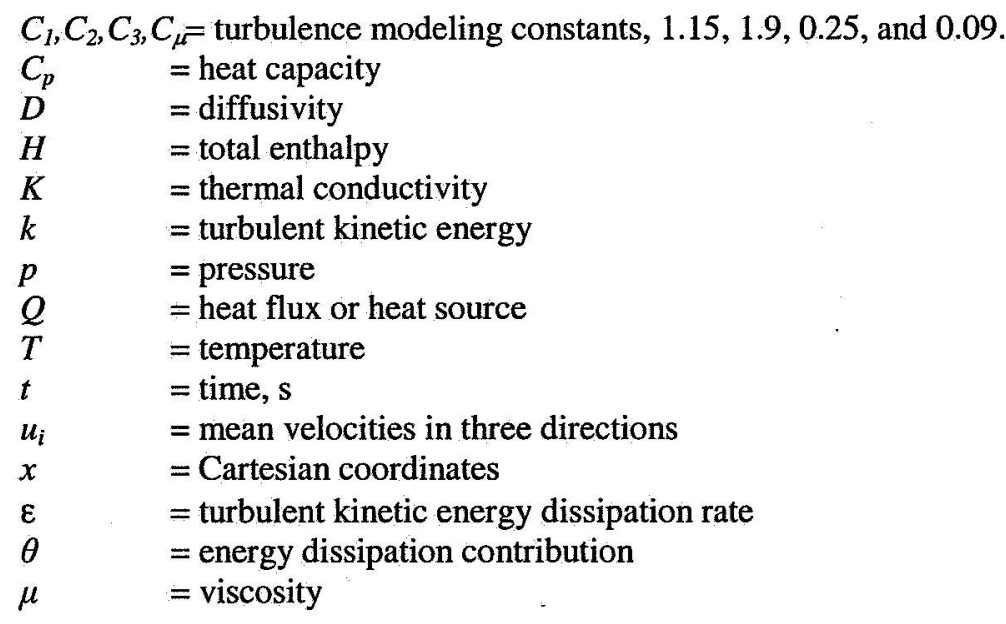

\footnotetext{
*Technical Assistant, ER43, Thermal and Combustion Analysis Branch, Propulsion Structure, Thermal, and Fluids Analysis Division, Senior Member AIAA.

${ }^{\dagger}$ Project Engineer, ER43, Thermal and Combustion Analysis Branch.

¥ Project Engineer, ER12, Propulsion Research Laboratory, Senior Member AIAA.

${ }^{\S}$ Project Engineer, ER12, Propulsion Research Laboratory, Associate Fellow AIAA.

${ }^{* *}$ President, Engineering Sciences, Inc., Senior Member AIAA.
} 


$$
\begin{array}{ll}
\mu_{t} & =\text { turbulent eddy viscosity }\left(=\rho \mathrm{C}_{\mu} \mathrm{k}^{2} / \varepsilon\right) \\
\Pi I & =\text { turbulent kinetic energy production } \\
\rho & =\text { density } \\
\sigma & =\text { turbulence modeling constants } \\
\tau & =\text { shear stress } \\
\omega & =\text { chemical species production rate }
\end{array}
$$

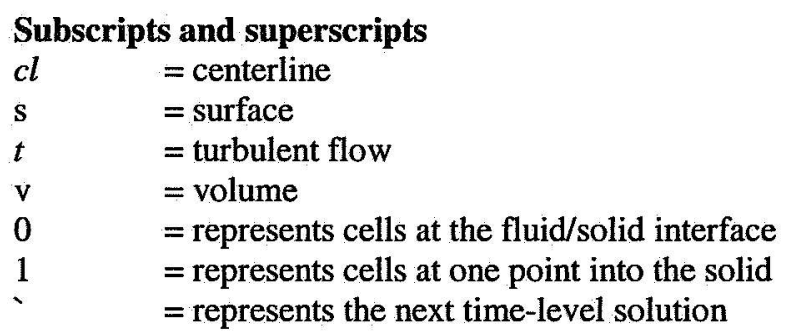

\section{Introduction}

$\mathrm{N}$ uclear thermal propulsion (NTP) may open up the solar system to far broader and faster exploration than is now possible with chemical propulsion. The feasibility of solid-core NTP systems was established by extensive testing in the Rover/NERVA programs and the technical merits of NTP have been identified and summarized. ${ }^{1}$ The basic concept involves a reactor core consisting of several hundred flow elements that are used to heat a low molecular weight gas, such as hydrogen. Thrust is generated as the hot hydrogen expands through a high expansion ratio nozzle. The need to push flow element temperature to extremes in order to maximize performance intensifies the possibility of material failures. ${ }^{2}$ For example, the mid-section corrosion was named after the materials at the mid-section of the flow element was found cracked after tests. ${ }^{2}$ In order to develop candidate high temperature materials that would be compatible with the hot-hydrogen environment of a high performance solid core NTP engine, new materials for flow elements need to be researched and tested. And accompanying computational methodology needs to be developed for analysis of test results in order to understand the contributing factors that lead to cracked materials, and to develop steps to improve the materials and operating procedures, so as to avoid future component failures.

In this work, a computational conjugate heat transfer methodology was developed to study the effect of thermal conductivity on thermal gradient inside a cylindrical specimen in a hot hydrogen environment, paralleling a hot hydrogen materials test effort that will test new materials inside an arc-heater based environments simulator. ${ }^{3}$ The computational methodology development is achieved by implementing a solid heat transfer analysis onto an existing computational fluid dynamics (CFD) methodology - an Unstructured-grid Navier-Stokes Internal-external CFD Code (UNIC), and by simulating the heat transfer characteristics of a cylindrical pellet made of materials used in legacy tests that experienced difficulties, ${ }^{2}$ heated by impinging hot hydrogen jet inside the arc-heater environments tester. Numerical procedures pertinent to conjugate heat transfer and physical properties germane to those legacy materials were implemented. The conjugate heat transfer implementation was anchored with a standard solid heat transfer code. The simulations of the heat transfer characteristics of the legacy materials were conducted for steadystate, impulsive start, and a $10 \mathrm{~s}$ linear ramp start conditions.

\section{Computational Methodology}

\section{A. Computational Fluid Dynamics}

The CFD methodology is based on a multi-dimensional, finite-volume, viscous, chemically reacting, unstructured grid, and pressure-based formulation. Time-varying transport equations of continuity, species continuity, momentum, total enthalpy, turbulent kinetic energy, and turbulent kinetic energy dissipation were solved using a time-marching sub-iteration scheme and are written as:

$$
\frac{\partial \rho}{\partial t}+\frac{\partial}{\partial x_{j}}\left(\rho u_{j}\right)=0
$$




$$
\begin{aligned}
& \frac{\partial \rho \alpha_{i}}{\partial t}+\frac{\partial}{\partial x_{j}}\left(\rho u_{j} \alpha_{j}\right)=\frac{\partial}{\partial x_{j}}\left[\left(\rho D+\frac{\mu_{t}}{\sigma_{\alpha}}\right) \frac{\partial \alpha_{i}}{\partial x_{j}}\right]+\omega_{i} \\
& \frac{\partial \rho u_{i}}{\partial t}+\frac{\partial}{\partial x_{j}}\left(\rho u_{j} u_{i}\right)=-\frac{\partial p}{\partial x_{i}}+\frac{\partial \tau_{i j}}{\partial x_{j}} \\
& \frac{\partial \rho H}{\partial t}+\frac{\partial}{\partial x_{j}}\left(\rho u_{j} H\right)=\frac{\partial p}{\partial t}+Q_{r}+\frac{\partial}{\partial x_{j}}\left(\left(\frac{K}{C_{p}}+\frac{\mu_{t}}{\sigma_{H}}\right) \nabla H\right)+\frac{\partial}{\partial x_{j}}\left(\left(\left(\mu+\mu_{t}\right)-\left(\frac{K}{C_{p}}+\frac{\mu_{t}}{\sigma_{H}}\right)\right) \nabla\left(v^{2} / 2\right)\right)+\theta \\
& \frac{\partial \rho k}{\partial t}+\frac{\partial}{\partial x_{j}}\left(\rho u_{j} k\right)=\frac{\partial}{\partial x_{j}}\left[\left(\mu+\frac{\mu_{t}}{\sigma_{k}}\right) \frac{\partial k}{\partial x_{j}}\right]+\rho(\Pi-\varepsilon) \\
& \frac{\partial \rho \varepsilon}{\partial t}+\frac{\partial}{\partial x_{j}}\left(\rho u_{j} \varepsilon\right)=\frac{\partial}{\partial x_{j}}\left[\left(\mu+\frac{\mu_{t}}{\sigma_{\varepsilon}}\right) \frac{\partial \varepsilon}{\partial x_{j}}\right]+\rho \frac{\varepsilon}{k}\left(C_{1} \Pi-C_{2} \varepsilon+C_{3} \Pi^{2} / \varepsilon\right)
\end{aligned}
$$

A predictor and corrector solution algorithm was employed to provide coupling of the governing equations. A second-order central-difference scheme was employed to discretize the diffusion fluxes and source terms. For the convective terms, a second-order upwind total variation diminishing difference scheme was used. To enhance the temporal accuracy, a second-order backward difference scheme was employed to discretize the temporal terms. Details of the numerical algorithm can be found in Ref's 4-8.

An extended $k-\varepsilon$ turbulence model ${ }^{9}$ was used to describe the turbulence. A modified wall function approach was employed to provide wall boundary layer solutions that are less sensitive to the near-wall grid spacing. Consequently, the model has combined the advantages of both the integrated-to-the-wall approach and the conventional law-of-the-wall approach by incorporating a complete velocity profile and a universal temperature profile $^{\top}$. A 2-species, 1-reaction detailed mechanism ${ }^{10}$ was used to describe the hydrogen dissociation and recombination chemical kinetics.

\section{B. Computational Conjugate Heat Transfer in Solids}

The solid heat conduction equation is solved with the gas-side heat flux distributions as its boundary conditions. The solid heat conduction equation can be written as:

$$
\frac{\partial \rho C_{p} T}{\partial t}-\frac{\partial}{\partial x_{j}}\left(K \frac{\partial T}{\partial x_{i}}\right)=Q_{v}+Q_{s}
$$

where $Q_{v}$ and $Q_{s}$ represent source terms from volumetric and boundary contributions, respectively. The present conjugate heat transfer model solves the heat conduction equation for the solid blocks separately from the fluid equations. The fluid/solid interface temperature, which is stored at interior boundary points, is calculated using heat flux continuity condition. For solution stability and consistency, the fluid/solid interface boundary temperature is updated using the transient heat conduction equation (7). The heat conduction equation on the fluid-solid interface can be discretized as:

$$
\frac{1}{2} \rho C_{p} \frac{T_{0}^{\prime}-T_{0}}{\Delta t}=\frac{1}{\Delta x_{j}}\left(k \frac{T_{1}-T_{0}}{\Delta x_{j}}-Q_{s}\right)
$$

where $Q_{s}$ is the wall heat flux from the solid wall to the fluid, which has an opposite sign to that from the fluid to the solid. The $1 / 2$ factor on the left hand side of the above equation comes from the fact that only half of the solid cell is involved in the control volume. The following expression is obtained for updating the interface temperature.

$$
T_{0}{ }^{\prime}=(1-A) T_{0}+A T_{1}-B Q_{s}
$$

where 


$$
\begin{aligned}
& A=\frac{2 \Delta t K}{\rho C_{p} \Delta x_{j}^{2}} \\
& B=\frac{2 \Delta t}{\rho C_{p} \Delta x_{j}}
\end{aligned}
$$

Since Eq. (8) is in transient form, it is applicable to transient applications. For steady-state solutions, acceleration factor could be applied to force faster convergence of heat conduction in the solid. In this work, a multiplication factor of 10 is applied to $A$ and $B$ for steady-state solutions.

\section{Test Fixture Description}

The cylindrical specimen to be analyzed is inserted in a sample holder and placed in a test fixture designed for new materials development and testing ${ }^{3}$ in a separate effort, as shown in Fig. 1. The test fixture is directly mated to an arc-heater (not shown) that provides hot-hydrogen flow. Optical ports are fitted to allow realtime pyrometer and laser diagnostics measurements for material surface temperature and centerline gas temperature, respectively. The copper test chamber is water-cooled. A tungsten/rhenium shield which serves as a convection shield and a radiation shield, allows the hot hydrogen jet to impinge on the specimen with

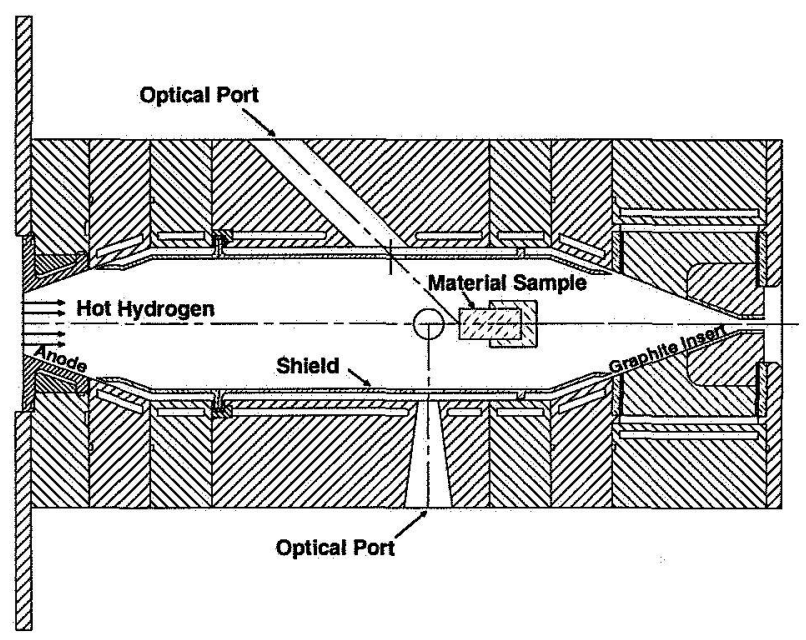

Fig 1. Test apparatus. maximum energy and minimum heat loss to the copper chamber. UNIC code was used to design the test fixture and details of the design analyses can be found in Ref. 3. In this work, the computational domain includes all the flow area as depicted in Figs 2 and 4, except for the arc-heater and the cooling channels. The effect of the cooling channels was represented as wall boundary condition with a separate calculation. ${ }^{3}$

\section{Computational Grid Generation}

Hybrid computational grids were generated using a software package GRIDGEN." Axisymmetric grids were used assuming the flow is symmetric about the axial axis. A series of grid verification studies using grid sizes ranging from 10,024 , to 19,448 were performed to determine the current grid size $(16,880){ }^{3}$ It was found during the grid study that the computed gas-solid interface temperature is most favorable when structuredgrid layers are present on both sides of the interface. Figure 2 shows such a grid layout where structured (rectangular) cells are used for the specimen and the region surrounding it. Structured cells are also used near wall boundaries, and are used for flow inlet and outlet boundaries, while the rest of the interior region is filled

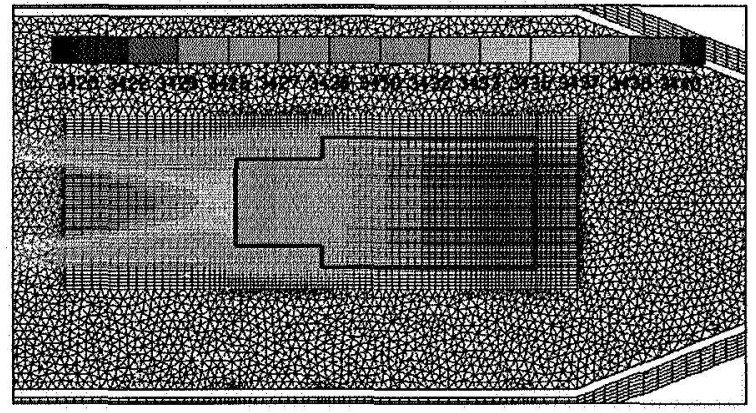

Fig. 2 Hybrid computational grid layout colored by typical computed temperature contours of hot hydrogen jet impinging on a rod-shaped specimen.

with unstructured (triangular) cells (mostly not shown). It can be seen from Fig. 2 that heat is being transferred from the impinging hot hydrogen jet to the specimen, and the computed specimen temperature contours exhibit an approximately one-dimensional behavior. 


\section{Boundary Conditions, thermal conductivities and run matrix}

No-slip condition was applied to the solid walls. Fixed mass flow rate boundary condition was used at the inlet, and mass conservation boundary condition was used at the exit. For a conservative analysis, the hot hydrogen enters the chamber at $3500 \mathrm{~K}$. A fixed temperature of $400 \mathrm{~K}$ was estimated for the chamber wall and the wall temperature of the shield facing the chamber wall was estimated to be $2600 \mathrm{~K}$, through separate one-dimensional heat transfer calculations. Adiabatic condition was applied to walls of opposing-side shield and flanges, and graphite insert (the convergent section). The hydrogen inlet mass flow rate was $10 \mathrm{~g} / \mathrm{s}$ and the chamber pressure was $35 \mathrm{~atm}$. A series of pre-calculations were performed to iterate the inlet temperature and species concentrations such that the inlet species concentrations correspond to a state of temperature at $3500 \mathrm{~K}$ for a nominal operating condition.

Table I shows the run matrix. Benchmark cases 1, 2, and 3 are steady-state runs with thermal conductivities of 50 , 80 , and 140 , respectively. The rest of the cases use variable thermal conductivities from measurement. Simulation cases 1,2 , and 3 are steady-state runs, while simulation cases 4,5 , and 6 are impulsive start runs, and simulation

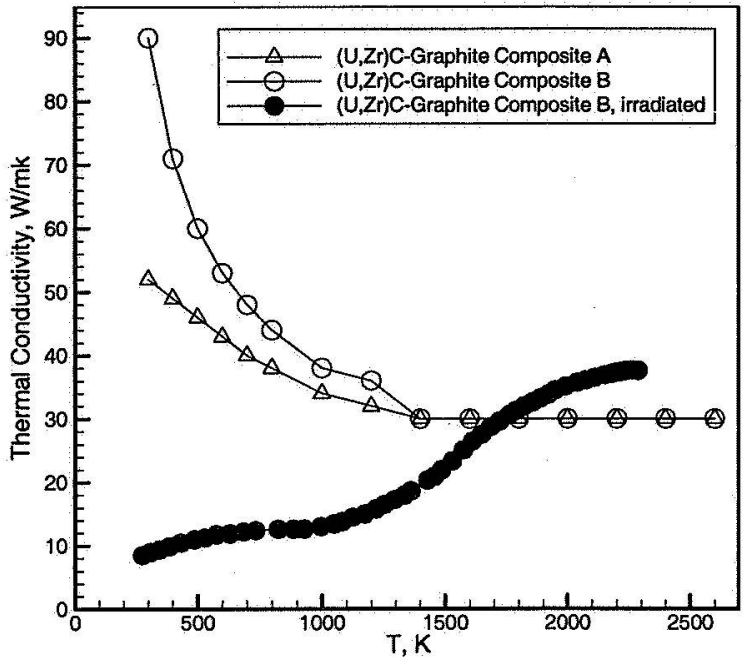

Fig. 3 Variable thermal conductivities for three specimens.

case 7 is a $10 \mathrm{~s}$ ramp start run. Composite $\mathrm{A}$ and $\mathrm{B}$, and irradiated composite $\mathrm{B}$ are the legacy materials tested in the 1970s. The irradiated Composite B is composite B material after test. The thermal conductivities of these specimens as function of temperatures ${ }^{2}$ are shown in Fig. 3. Composite B represents about the highest low temperature thermal conductivity that can be achieved that still retains desirable mechanical properties. The thermal conductivities of the irradiated composite B appear quite different from those of un-irradiated composite B, and the reason of which was speculated as a result of annealing that occurred during the time the measurements were being taken. ${ }^{2}$

Table I. Run matrix.

\begin{tabular}{|l|l|l|}
\hline Case & state & K \\
\hline Benchmark 1 & Steady & 50 \\
\hline Benchmark 2 & Steady & 80 \\
\hline Benchmark 3 & Steady & 140 \\
\hline Simulation 1 & Steady & Composite A \\
\hline Simulation 2 & Steady & Composite B \\
\hline Simulation 3 & Steady & Irradiated composite B \\
\hline Simulation 4 & Impulsive start & Composite A \\
\hline Simulation 5 & Impulsive start & Composite B \\
\hline Simulation 6 & Impulsive start & Irradiated composite B \\
\hline Simulation 7 & 10 s ramp start & Composite B \\
\hline
\end{tabular}

\section{Results and Discussion}


Figure 4 shows typical computed temperature, $\mathrm{H}$ (atom) concentration contours and streamlines under nominal steady-state test conditions, for the entire computational domain. It can be seen from the temperature contours that the entire thrust chamber is almost uniformly heated, except for the region between the shield and the cooled chamber wall. This is because the shield serves as both convection and radiation shields. Nevertheless, there is still some heat loss to the environment and the hot hydrogen jet temperature drops slightly as it impinges and wraps around the material specimen. When temperature decreases, $\mathrm{H}$ recombines to become $\mathrm{H}_{2}$. This is shown in the $\mathrm{H}$ contours where its concentration decreases as the hot jet approaches the sample. The computed streamlines show an expanding hot-hydrogen jet impinging on and flowing around the specimen, and later exhausting into the convergent exit section. A large recirculation zone appears in the divergent section of the chamber, while a small recirculation region forms behind the sample holder; both of which are strongly affected by turbulence. Note although the plots show a distinctively strong hot-hydrogen jet and two recirculated flow regions, the pressure and Mach number contours are fairly uniform inside the chamber (not shown), due to the largely low subsonic flow field and the protection of the shield. The flow becomes supersonic in the exit pipe.

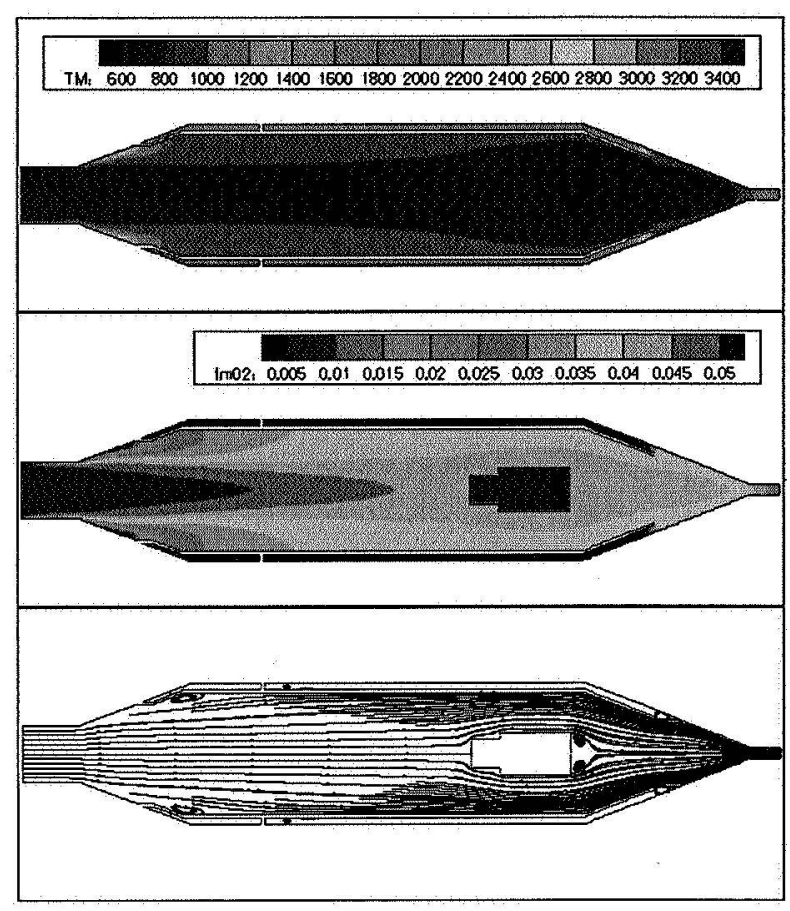

Fig. 4 From top to bottom, typical computed steady-state temperature and $\mathrm{H}$ Mass Fraction Contours and Streamlines.

\section{Steady-State Benchmark Analysis with Constant Thermal Properties}

Figure 5 shows a comparison of the computed steady-state centerline coupon temperatures, using conjugate heat transfer methodology developed in this work, with those obtained from a standard solid heat transfer code SINDA. ${ }^{12}$ Solutions for three constant thermal conductivities, 50, 80, and 140, measured at room temperature for flow element materials heat treated at 2800,3050 , and $3120 \mathrm{~K}$, respectively, ${ }^{2}$ were performed. The first iteration of SINDA solution was based on three arbitrarily chosen wall temperatures of 300,2500 , and 3400 $\mathrm{K}$, by assuming the final solid temperatures are between 300 and $3400 \mathrm{~K}$. It should be noted that these fixed wall temperatures imply three wall heat flux profiles. It can be seen that the UNIC computed centerline temperatures compared reasonably well with those obtained from the first SINDA iteration, and the maximum deviation is less than $10 \mathrm{~K}$. Based on the temperatures calculated in the first iteration, the second SINDA iteration was performed and the three input wall temperatures were narrowed to 3405,3410 , and $3413 \mathrm{~K}$. It can be seen that solutions of UNIC and those of second SINDA iteration are now very well compared, and the deviation is less than $2 \mathrm{~K}$.

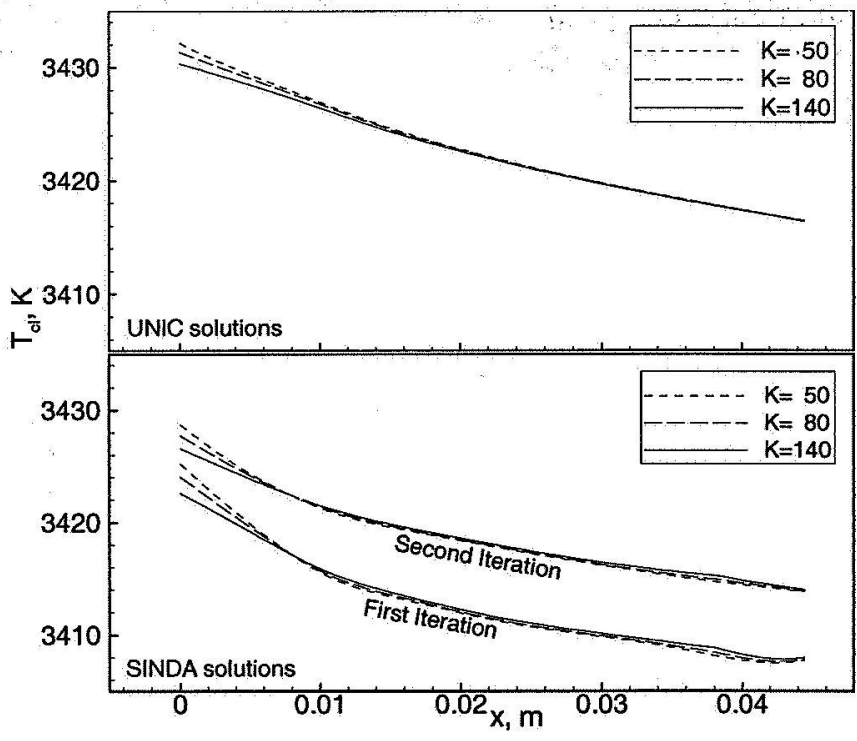

Fig. 5 A comparison of computed steady-state centerline specimen temperatures.

In summary, Fig. 5 demonstrates that the UNIC computed solid temperatures compare very well with those of a standard solid heat transfer code SINDA. Both UNIC and SINDA solutions exhibit similar behavior. For example, high thermal gradients occur near the stagnation point $(x=0)$. In addition, the lowest thermal conductivity $(k=50)$ gives the highest thermal gradient, while the highest thermal conductivity $(K=140)$ results in the lowest thermal gradient. 


\section{Analysis with Variable Thermal Properties}

\section{Steady-State Analysis}

Three constant thermal conductivities were used to perform benchmark analysis as mentioned above. For actual flow element materials such as Composite $A$, Composite B and irradiated Composite B, their conductivities varies with temperature, as shown in Fig. 3. Figure 6 shows the computed steady-state centerline temperatures for these three specimens, simulated as cylindrical pellets heated by hot hydrogen jet impingement as described in Fig. 1. It can be seen that the centerline temperature profiles for Composite $\mathrm{A}$ and Composite B overlap, while that for the irradiated Composite B deviate only slightly near the stagnation point $(x=0)$ and near $x=0.04$. This is because the cylindrical pellet is surrounded by hot hydrogen flow and the whole pellet is heated up to temperatures from about 3400 to $3425 \mathrm{~K}$, as shown in Figs. 2 and 4. At these temperature levels, the thermal conductivities are nearly constant $(30 \sim 35 \mathrm{~W} / \mathrm{mK})$, as shown in Fig. 3 .

The temperature scale in Fig. 6 is zoomed in, that is, the steady-state thermal gradients for all three specimens appear to be benign, implying that there was no high thermal gradient or high thermal stress for these pellets, during the steady-state heating by hot hydrogen. Although the set up of impingement heating of a short pellet (fig. 1) is not intended to explain the mid-section thermal anomaly of a internally heated, full-length flow element, rather a simpler way to isolate the physics. With the range of thermal conductivities shown in Fig. 3, it is speculated however, that there should be stronger thermal gradients during the transient impingement heating process. That possibility is investigated by performing three impulsive and one linear $10 \mathrm{~s}$ ramp startup computations, as discussed in the following.

\section{Transient Analysis - Impulsive Start}

Three impulsively started transient conjugate heat transfer computations were performed for the three specimens with the variable thermal conductivities as described in Fig. 3. Hydrogen gas was assumed to be in the tester initially at initial temperature and pressure of $300 \mathrm{~K}$ and $1 \mathrm{~atm}$, respectively. The initial pellet temperature was assumed to be at $300 \mathrm{~K}$. For the most conservative case, hot hydrogen was assumed to enter the tester impulsively at $3500 \mathrm{~K}$ and $35 \mathrm{~atm}$, respectively, meaning the inlet hydrogen gas was heated to $3500 \mathrm{~K}$ and pressurized to $35 \mathrm{~atm}$ instantly by the arc heater at time zero.

As mentioned in the last section, it was anticipated that impulsive would create high thermal gradient, or thermal shock in the solids. Figure 7 shows the computed transient centerline temperature profiles for composite A. It can be seen that unlike the steady-state profile, strong thermal gradients occur at both ends of the pellet, where the impingement end or the stagnation point end $(x=0)$ encounters the strongest thermal gradient throughout most of the transient heating process, especially during the 3 to $7 \mathrm{~s}$ period. During that time period, it can be seen that the center core had a difficult time to get heated up from 1600 to $1800 \mathrm{~K}$, resulting in strong thermal gradients at the two ends. This phenomenon is

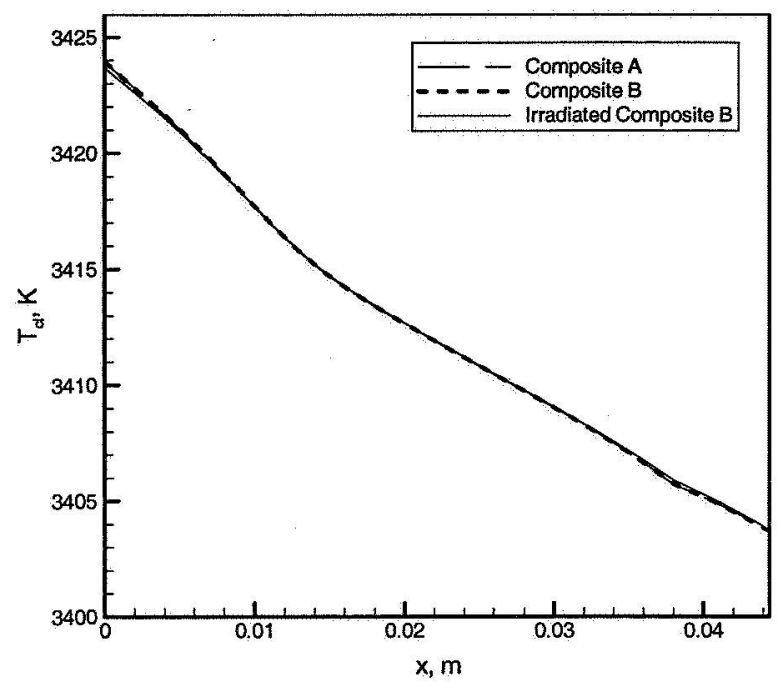

Fig. 6 Computed steady-state centerline temperatures for three hypothetical specimens.

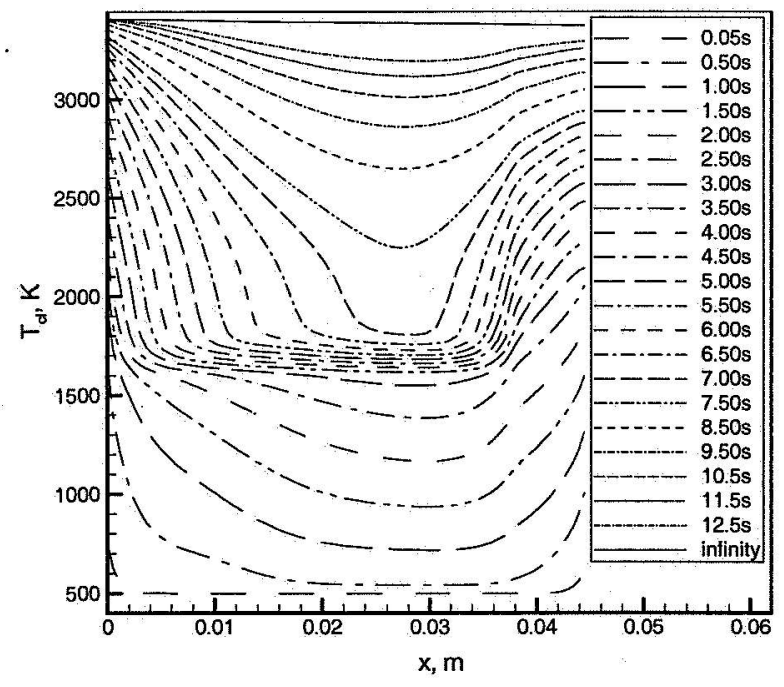

Fig. 7 Computed cylindrical specimen centerline temperature history for Composite A during an impulsive start. 
strongly correlated to the low thermal conductivity at high temperatures for composite A, as shown in Fig. 3 .

Figures 8 and 9 show the computed transient temperature profiles for composite B and irradiated composite B, respectively. It can be seen that except in the early heating period $(0.05 \sim 1.5 \mathrm{~s})$ when the irradiated composite B shows stronger thermal gradient than that of composite B, both show similarly strong thermal gradients at the two ends when

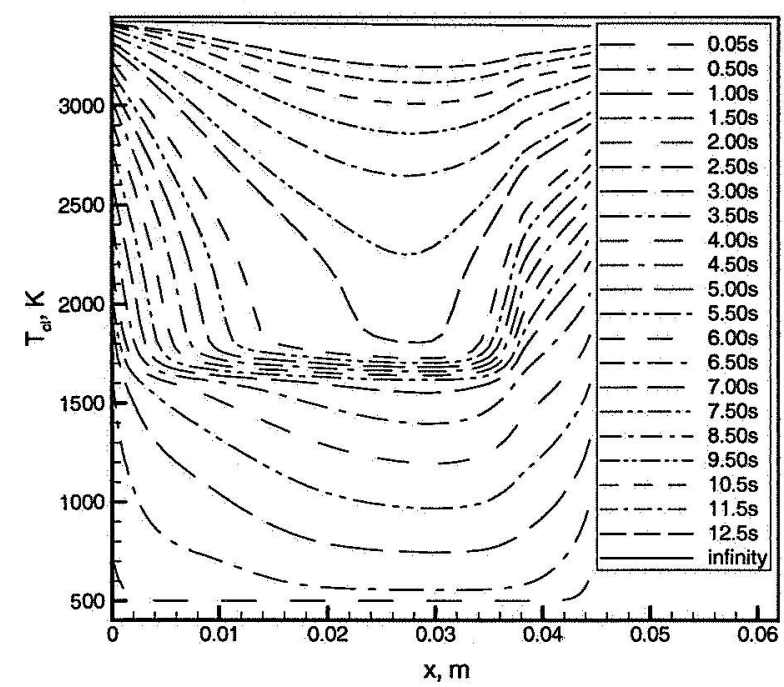

Fig. 8 Computed cylindrical specimen centerline temperature history for Composite B during an impulsive start.

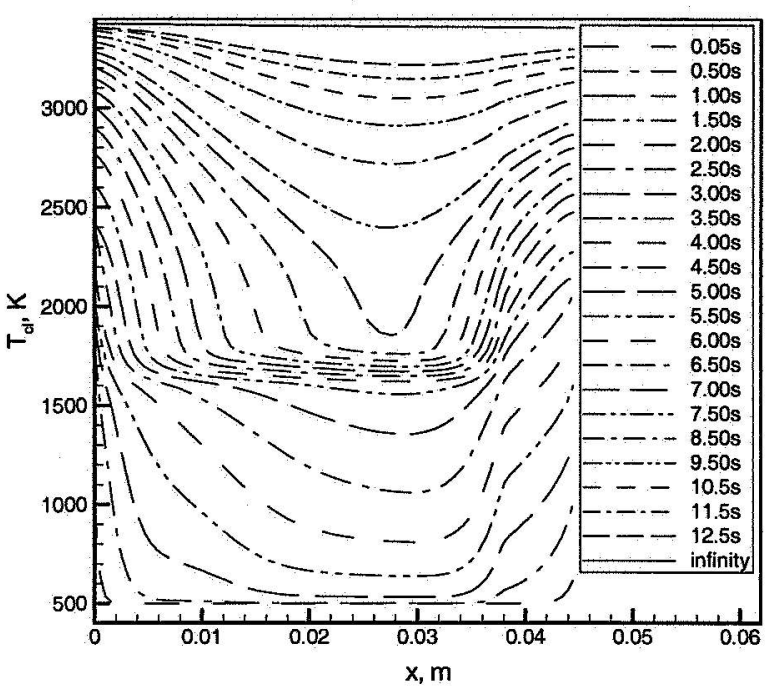

Fig. 9 Computed cylindrical specimen centerline temperature history for irradiated Composite B during an impulsive start.

the central core reaches $1600 \mathrm{~K}$. In fact, during that time period, the transient temperature profiles of composite B and irradiated composite B look similar to those of composite A (Fig. 7).

The similarity in the occurrence of high thermal gradient during the $3 \sim 7 \mathrm{~s}$ time period for composite $\mathrm{A}$ and $\mathrm{B}$, and irradiated composite $\mathrm{B}$ correlates to the fact that the thermal conductivity of all three specimen converge to a similarly low value as temperature exceeds $1,600 \mathrm{~K}$, even though their individual thermal conductivity profiles look quite different in lower temperatures. Figures 7,8 , and 9 demonstrate that the thermal wave propagation inside the cylindrical pellet is a strong function of the characteristics of its thermal conductivity. In addition, it could be inferred that for other specimens with completely different thermal conductivity characteristics, different thermal transient histories would have been computed. Figures 7, 8, and 9 also show that, for the three cylindrical flow element specimens studied, it takes more than $12.5 \mathrm{~s}$ to heat the pellets up, using a impulsive start.

\section{Transient Analysis - 10 s Ramp Start}

The $10 \mathrm{~s}$ ramp start assumes the hydrogen gas inlet temperature and pressure are brought linearly to $3,500 \mathrm{~K}$ and $35 \mathrm{~atm}$, from $300 \mathrm{~K}$ and $1 \mathrm{~atm}$, respectively, during a $10 \mathrm{~s}$ period. This means that the leading edge of the cylindrical pellet does not see the final temperature until the $10^{\text {th }} \mathrm{s}$, comparing to sensing the final temperature at the start command of the impulsive start. Only the effect of thermal conductivity characteristics of Composite B was investigated, since the impulsive start analyses show that the transient behaviors of all three specimens are similar at high temperatures. Figure 10 shows the computed transient centerline temperature history for Composite B during the $10 \mathrm{~s}$ ramp start. It can be seen that from 0.05 to $8.5 \mathrm{~s}$, the thermal gradients are fairly benign at both ends of the

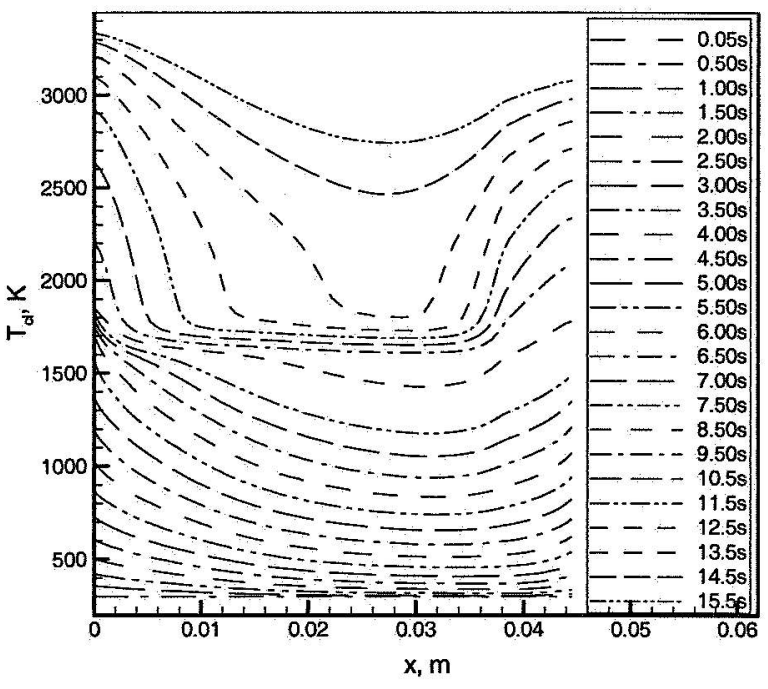

Fig. 10 Computed cylindrical specimen centerline temperature history for composite B during a $10 \mathrm{~s}$ ramp start. 
cylindrical pellet. At $9.5 \mathrm{~s}$, as the center core temperature reaches $1,600 \mathrm{~K}$, higher thermal gradients again start to show. Hence, the thermal conductivity effect from Fig. 3 still dominates in the $10 \mathrm{~s} \mathrm{ramp} \mathrm{start,} \mathrm{even} \mathrm{thought} \mathrm{the} \mathrm{high} \mathrm{thermal}$ gradient occurs about $6.5 \mathrm{~s}$ later than those of the impulsive starts. The total heat up time is more than $15.5 \mathrm{~s}$.

\section{Conclusion}

A computational conjugate heat transfer methodology was developed, to study the heat transfer effect of a hot hydrogen jet impinging on a cylindrical pellet made of hypothetical thermal nuclear rocket flow element materials, as a first step towards developing a multiple physics, thermo-fluid computational methodology to predict environments for hypothetical solid-core, nuclear thermal engine thrust chamber and components. This is accomplished by implementing a solid heat transfer procedure onto an existing computational fluid dynamics formulation. The solid heat transfer module was anchored and the computed pellet centerline temperatures compare very well with those of a standard solid heat transfer code. Analyses were then performed for hypothetical cylindrical pellet using variable thermal conductivities of three legacy flow element materials. Under the assumptions made in this study, it was found that high thermal gradients occur during the transient heating processes and not the steady-state heating processes. High thermal gradients occur at both ends of the pellet, especially near the impingement point. The transient heating behavior inside these rod-shaped specimens depends solely on their thermal conductivity characteristics. For the three specimens studied, since the thermal conductivities of all three specimen converge to a constant low value as temperatures exceed $1,600 \mathrm{~K}$, high thermal gradient starts to form at both ends of the pellet when the center core temperatures reaches $1,600 \mathrm{~K}$, during either the impulsive or $10 \mathrm{~s}$ ramp start process.

\section{Acknowledgments}

This study was partially supported by a Nuclear Systems Office task entitled "Multiphysics Thrust Chamber Modeling" and by a MSFC Internal Research and Development focus area task entitled "Hot-Hydrogen Materials and Component Development." Thermal properties provided by Panda Binayak, Robert.Hickman, and Bill Emrich are acknowledged.

\section{References}

${ }^{1}$ Koenig, D.R., "Experience Gained from the Space Nuclear Rocket Program (Rover)," LA-10062-H, Los Alamos National Laboratory, Los Alamos, New Mexico, 1986.

${ }^{2}$ Lyon, L.L., "Performance of (U,Zr)C-Graphite (Composite) and of (U,Zr)C (Carbide) Fuel Elements in the Nuclear Furnace 1 Test Reactor," LA-5398-MS, Los Alamos Scientific Laboratory, Los Alamos, New Mexico, 1973.

${ }^{3}$ Wang, T.-S., Foote, J., and Litchford, R., "Multiphysics Thermal-Fluid Design Analysis of a Non-Nuclear Tester for HotHydrogen Material Development," Space Technology and Applications International Forum (STAIF-2006), Albuquerque, NM, Fe. 12-16, 2006, American Institute of Physics Proceedings, edited by El-Genk, M.S., Melville, N.Y., Vol. 813, 2006, pp. 537544.

${ }^{4}$ Chen, Y.-S., Liu, J., Zhang, S., and Mallapragada, P., “An Integrated Tool for Launch Vehicle Base-Heating Analysis," Final Report, NAS8-00002, Engineering Sciences, Inc., Huntsville, AL, 2001

${ }^{5}$ Chen. Y.-S., Zhang S., and Liu, J., "Stage Separation Performance Analysis Project," Final Report, H-34345D, Engineering Sciences, Inc., Huntsville, AL, 2002.

${ }^{6}$ Wang, T.-S., Chen, Y.-S., Liu, J., Myrabo, L.N., and Mead, F.B. Jr., "Advanced Performance Modeling of Experimental Laser Lightcraft," Journal of Propulsion and Power, Vol. 18, No. 6, 2002, pp. 1129-1138.

${ }^{7}$ Wang, T.-S., "Multidimensional Unstructured-Grid Liquid Rocket Engine Nozzle Performance and Heat Transfer Analysis," Journal of Propulsion and Power, Vol. 22, No. 1, 2005, pp. 78-84.

${ }^{8}$ Wang, T.-S., "Transient 3-D Analysis of Nozzle Side Load in Regeneratively Cooled Engines," AIAA Paper 2005-3942, $41^{\text {st }}$ AIAA/ASME/SAE/ASEE Joint Propulsion Conference, Tucson, Arizona, 2005.

${ }^{9}$ Chen, Y.-S., and Kim, S. W., "Computation of Turbulent Flows Using an Extended k- $\varepsilon$ Turbulence Closure Model," NASA CR-179204, 1987.

${ }^{10}$ Wang, T.-S., "Thermophysics Characterization of Kerosene Combustion," Journal of Thermophysics and Heat Transfer, Vol. 15, No. 2, 2001, pp.140-147.

${ }^{11}$ Steinbrenner, J.P., Chawner, J.R., and Fouts, C., "Multiple Block Grid Generation in the interactive Environment," AIAA Paper 90-1602, June 1990.

${ }^{12}$ Gaski, J., "The Systems Improved Numerical Differencing Analyzer (SINDA) Code - a User's Manual," Aerospace Corp., El Segundo, CA, Feb. 1986. 


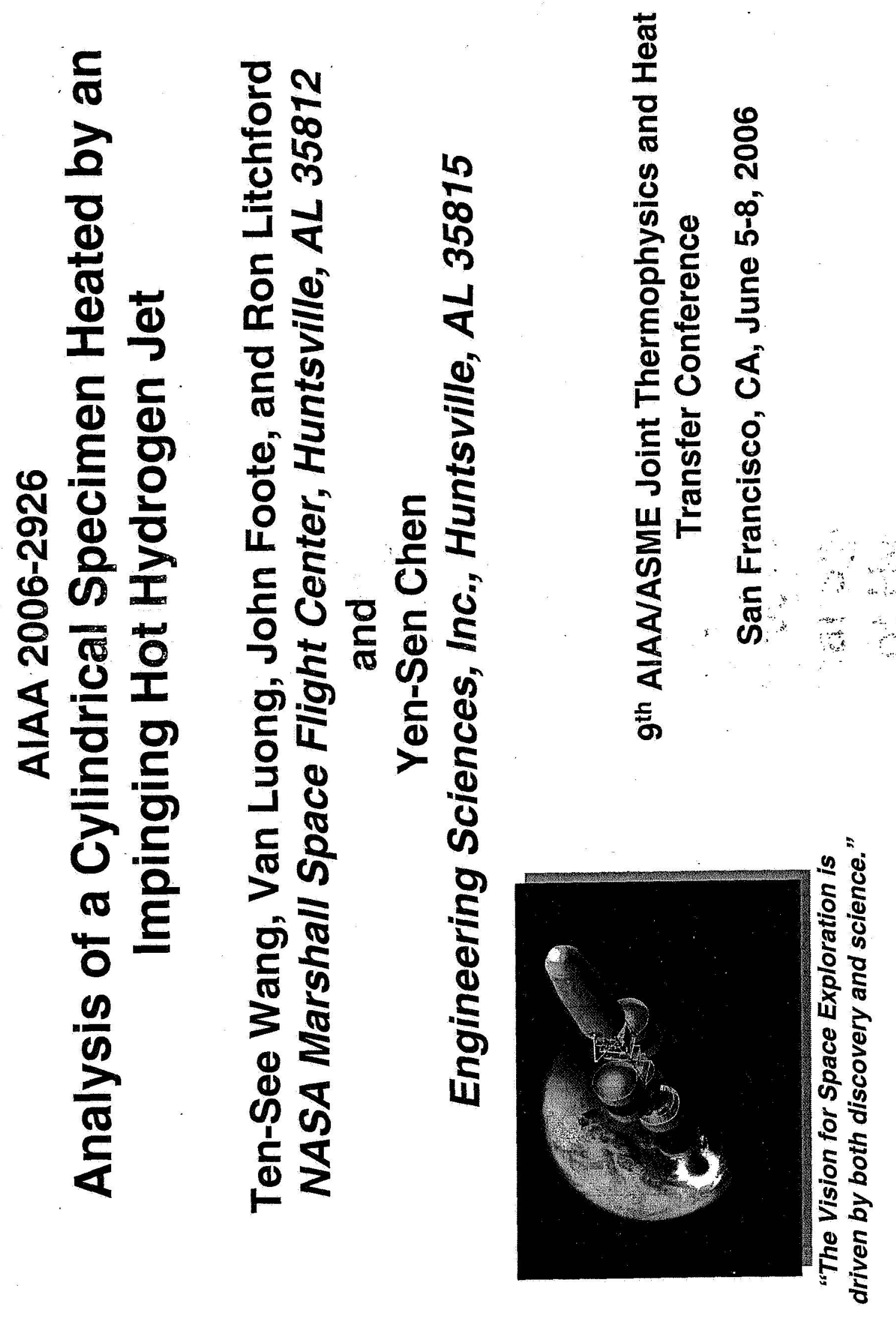




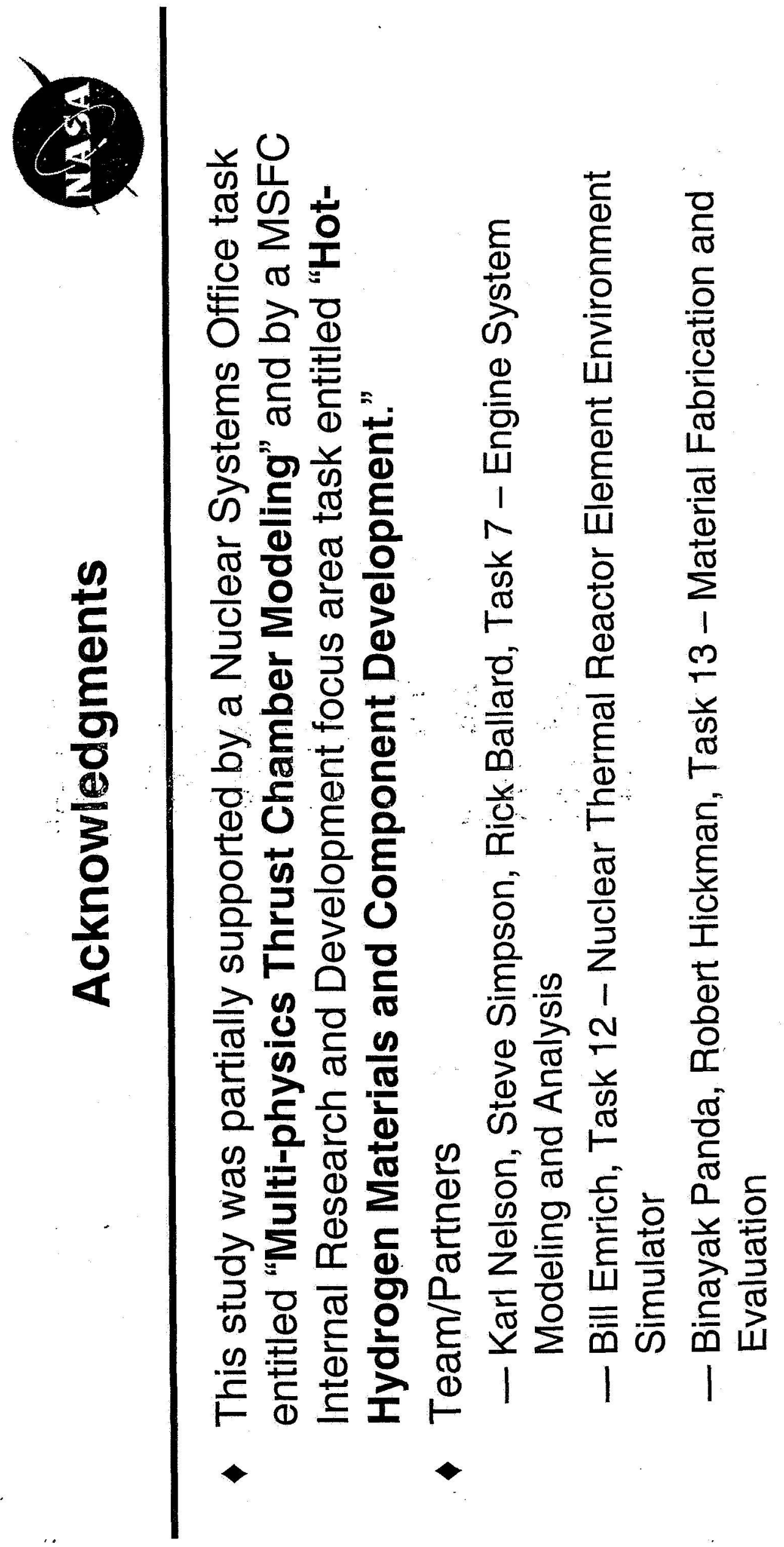




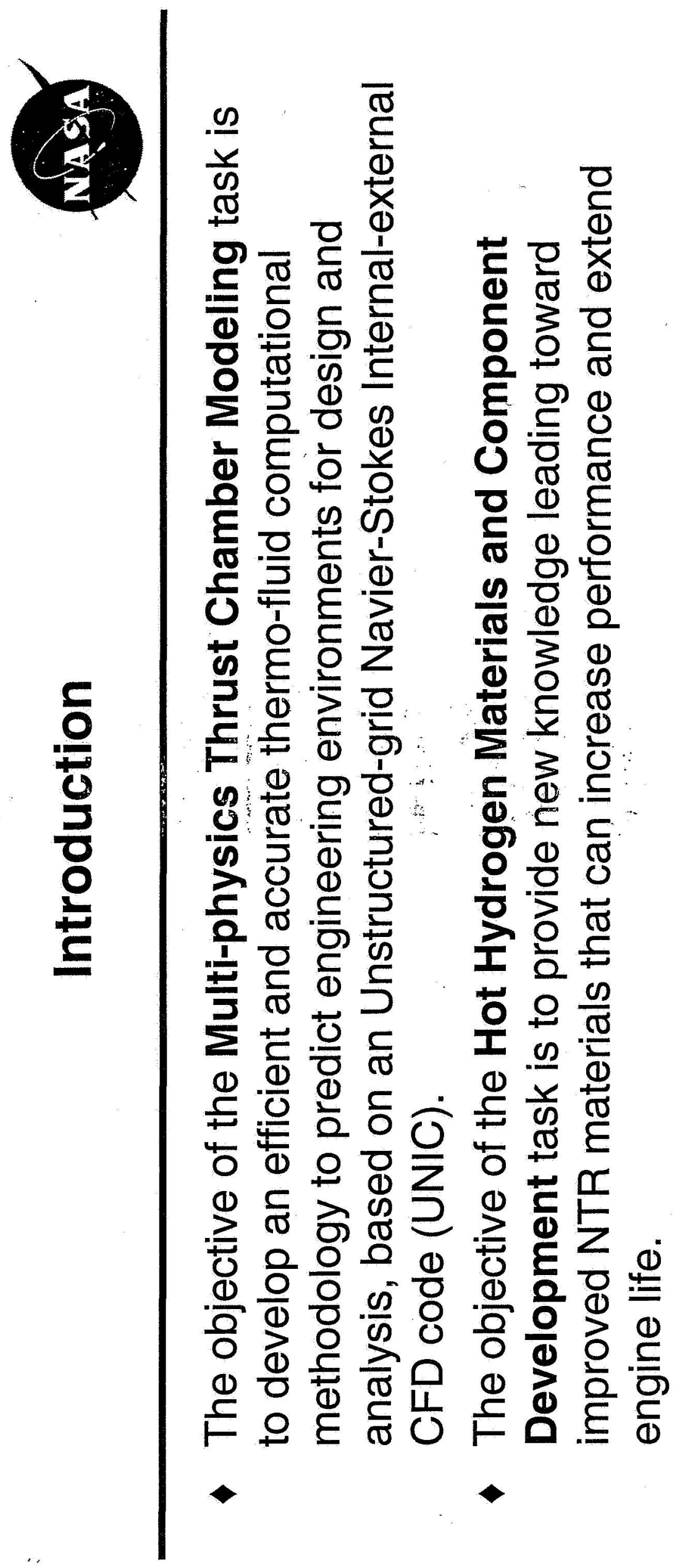



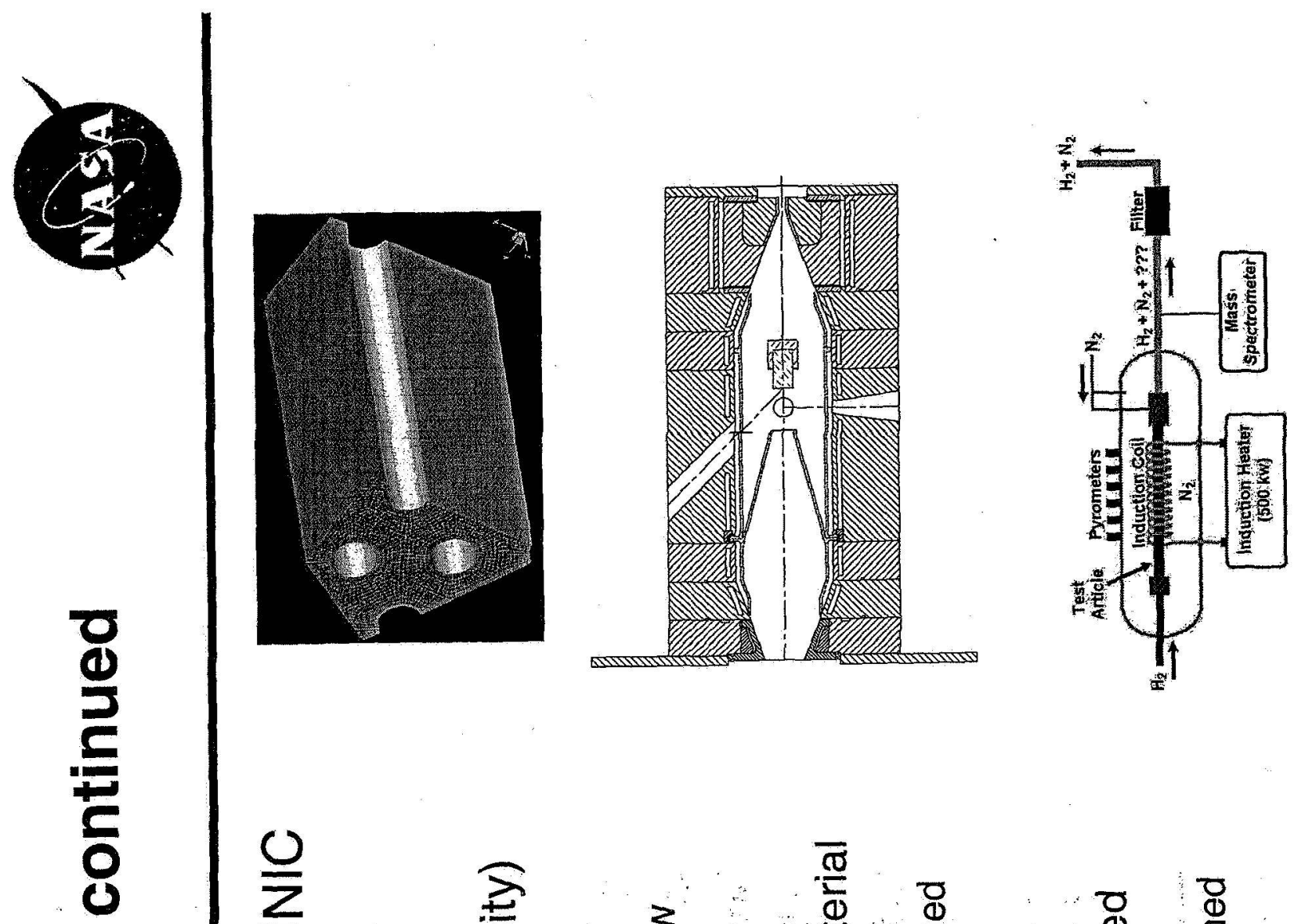

음

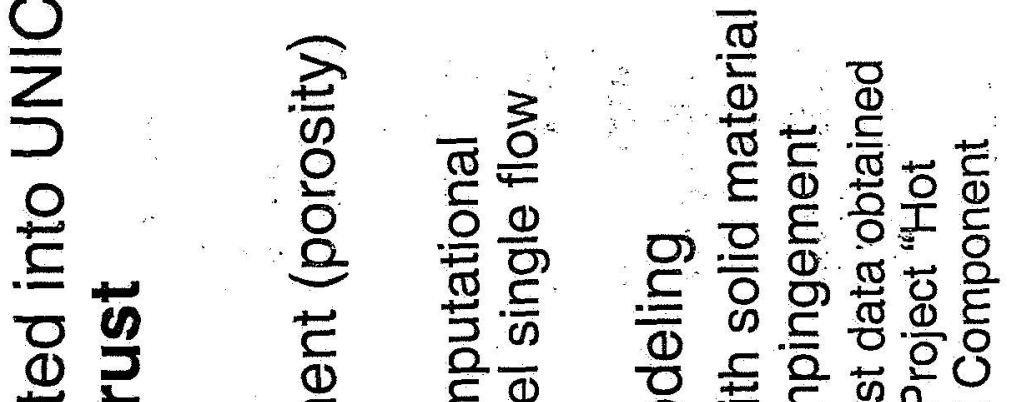

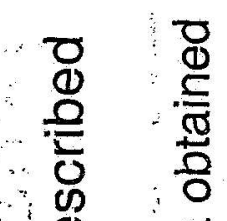

$\frac{\pi}{2}$

䒕ㄴ.

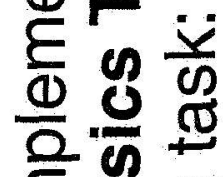

о

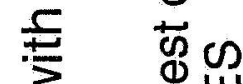

잉

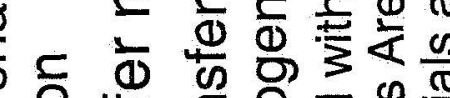

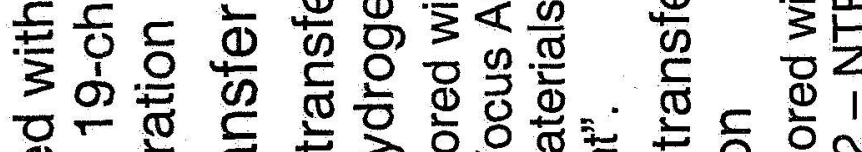

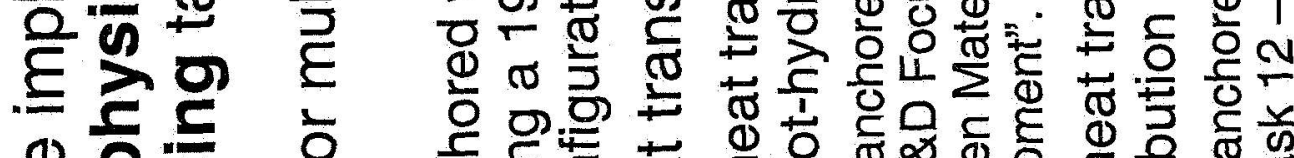

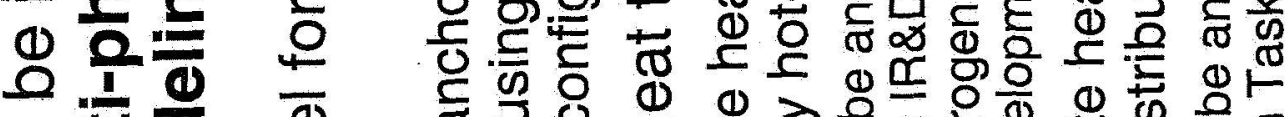

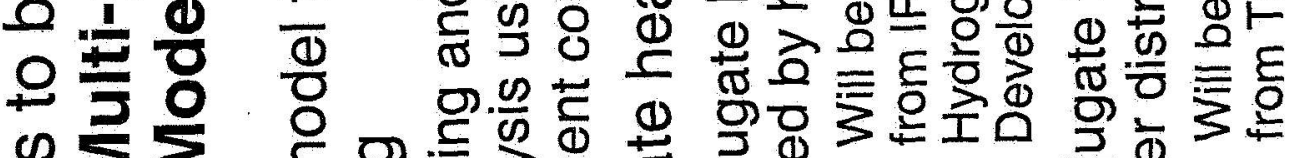

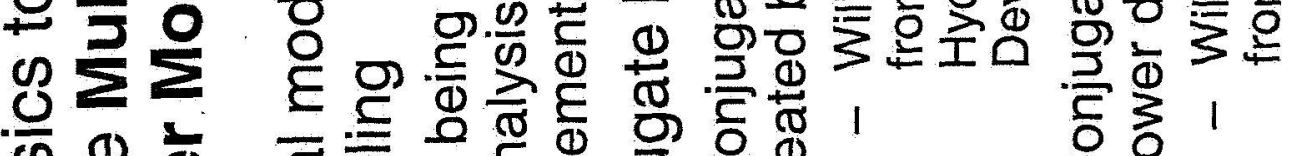

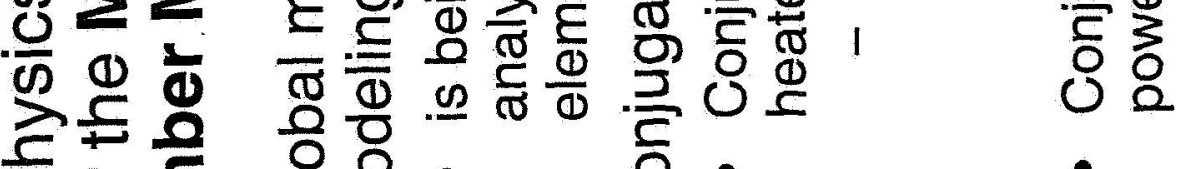

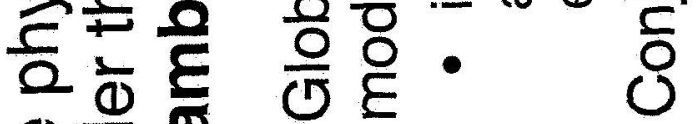

बक क ज

F号 । 


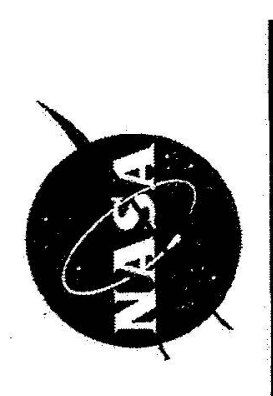

(1)

(1)

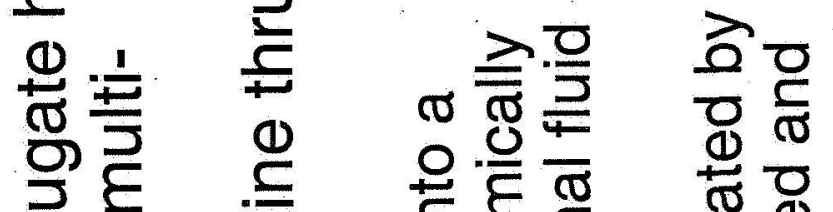

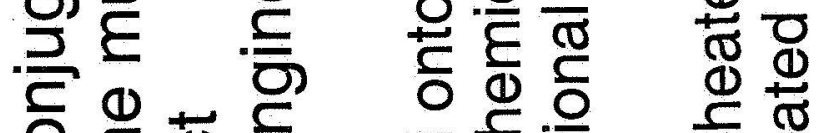

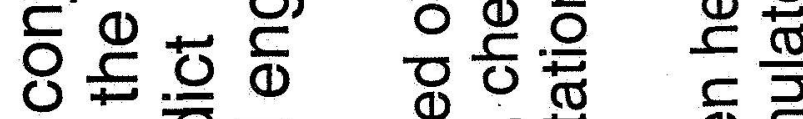

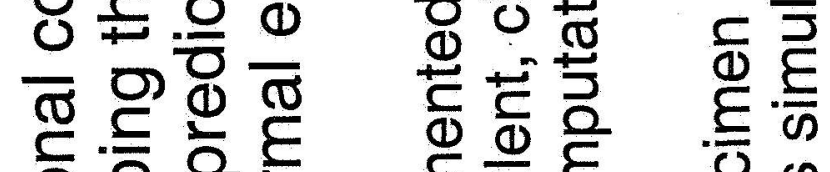

응 0 잉 ह

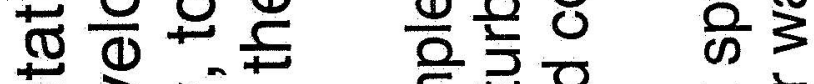

इ

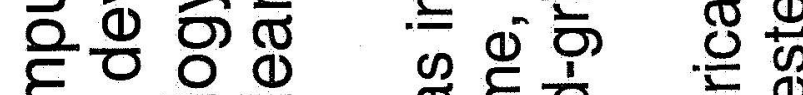

E ल $\frac{0}{0}$ ज

ชํำ

ช

0
0
0
0

$\frac{0}{0} \stackrel{1}{0}$

(1)

$3 \frac{2}{0} \quad$ 웡

ఏ

ํํㄴ롤

母 $\pm \equiv$

을

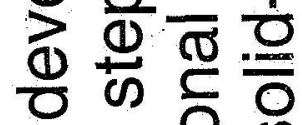

남

ส

os

$\equiv \frac{0}{\sigma}$

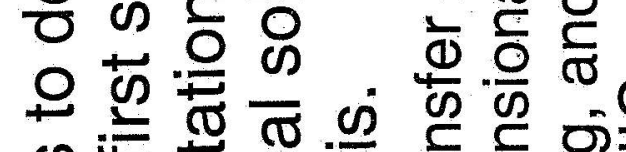

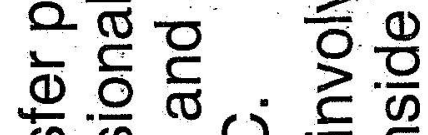

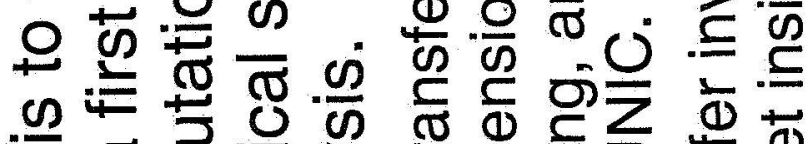

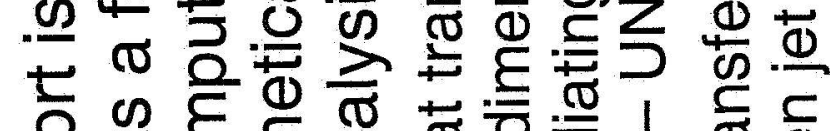

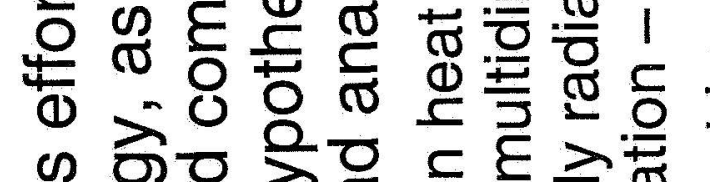

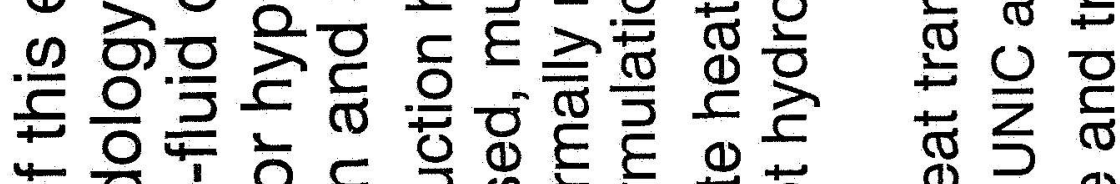

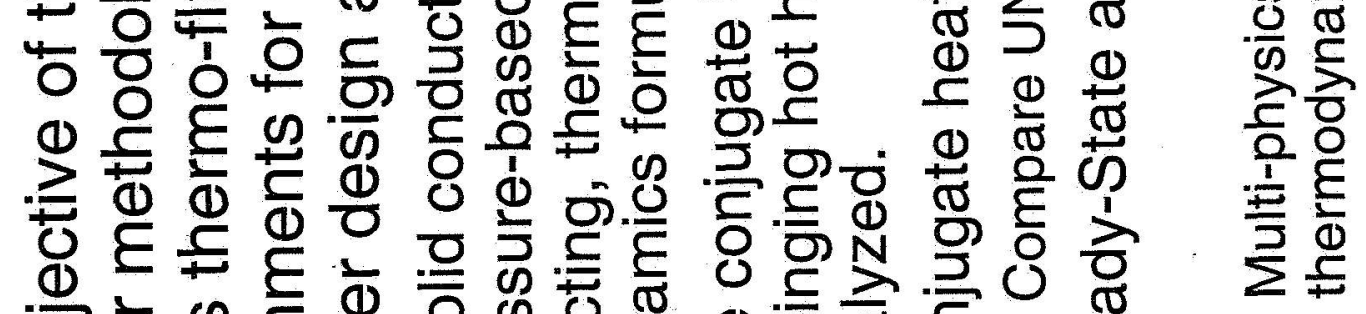

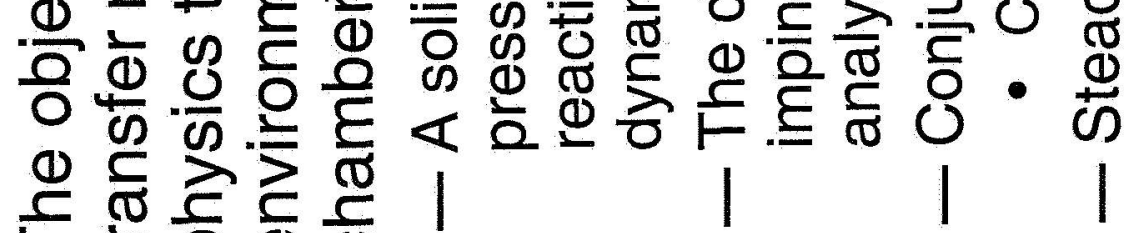




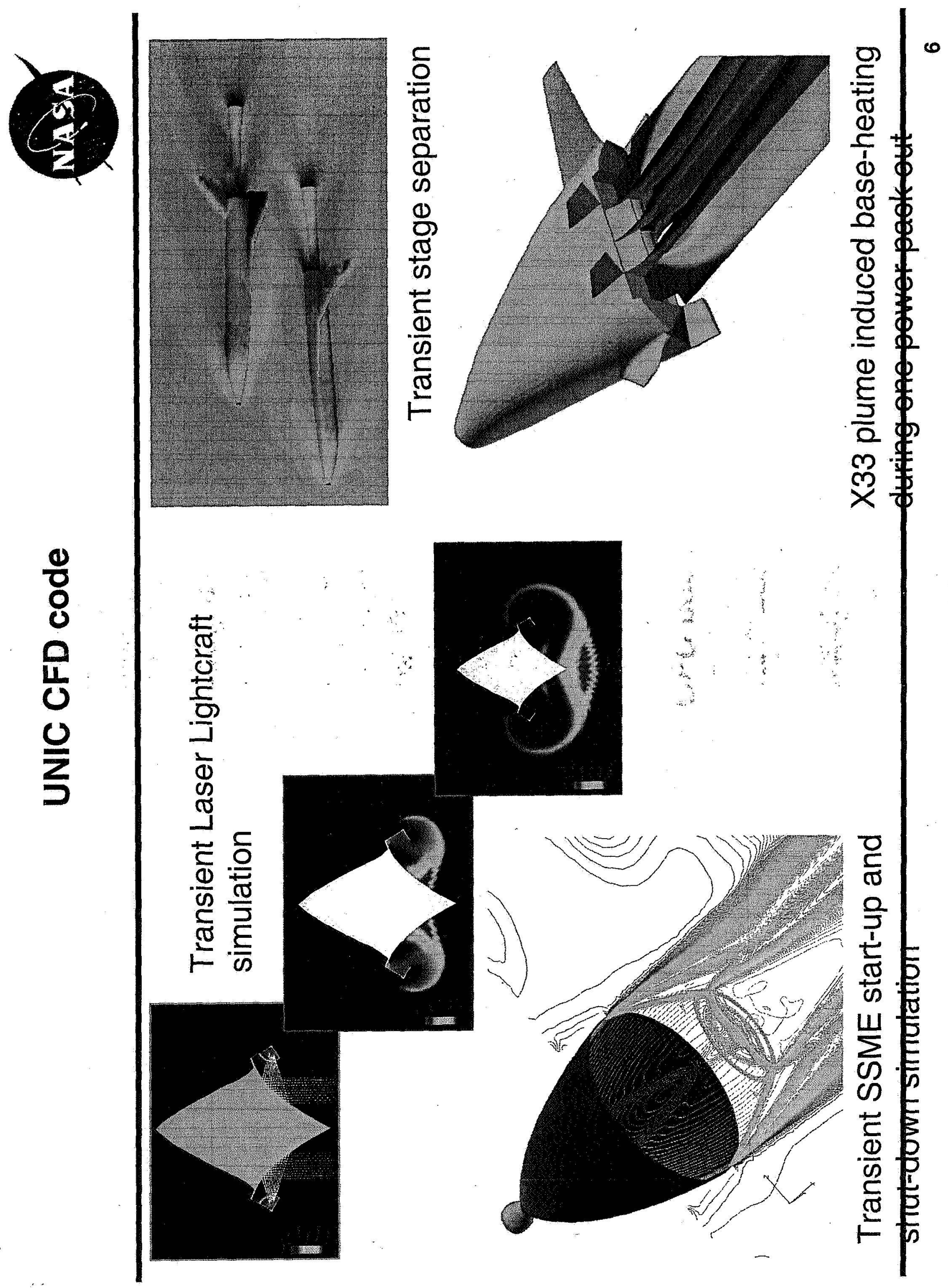




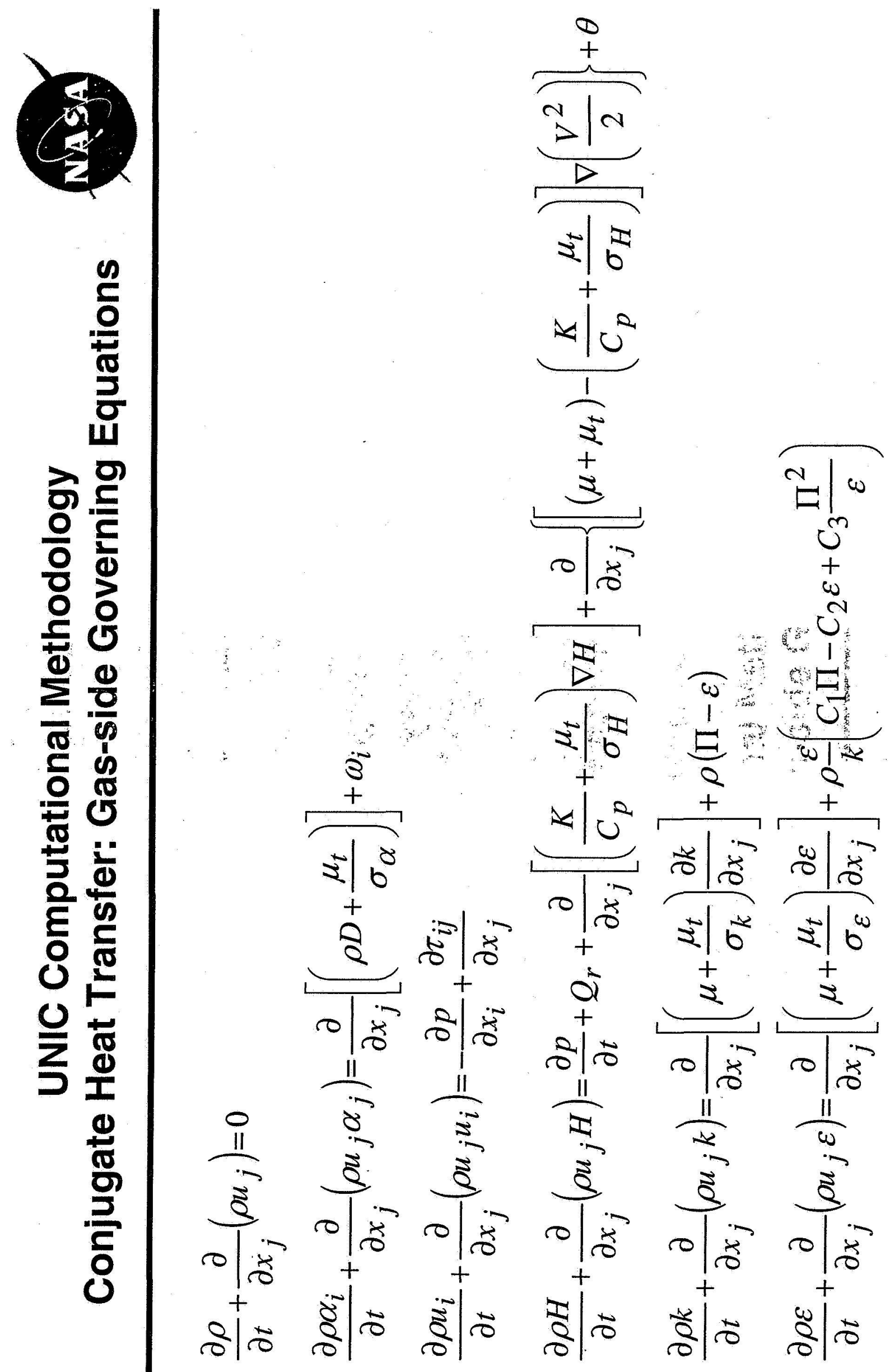




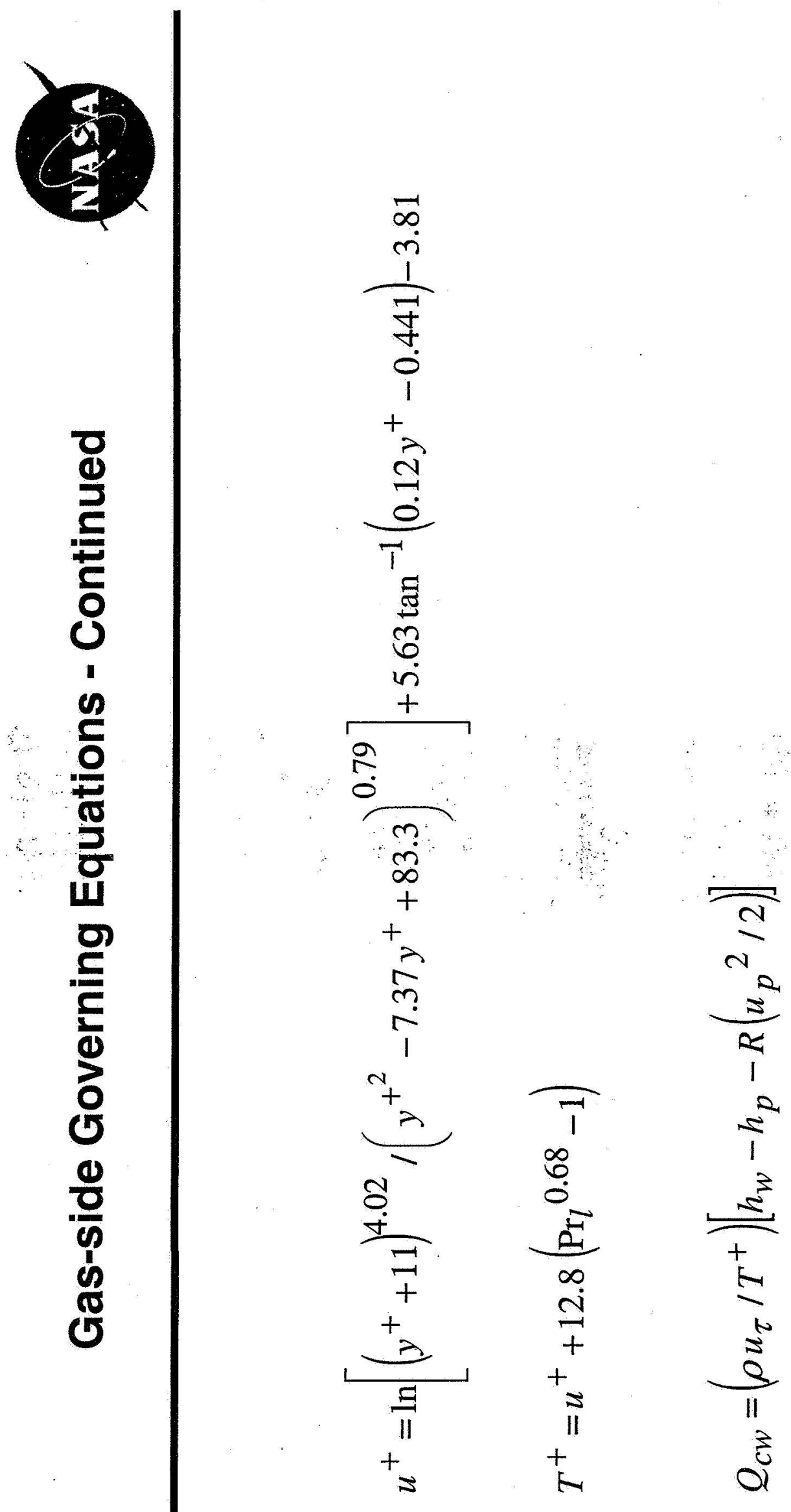




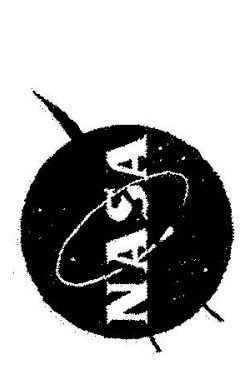

$\frac{0}{5}$

5

$\frac{0}{\frac{0}{0}}$

$\frac{5}{0}$

$0^{2}$

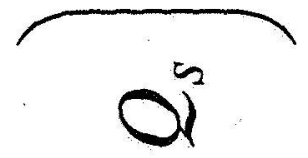

$\frac{0}{2}$

$\frac{\frac{5}{0}}{\frac{5}{0}}$

-

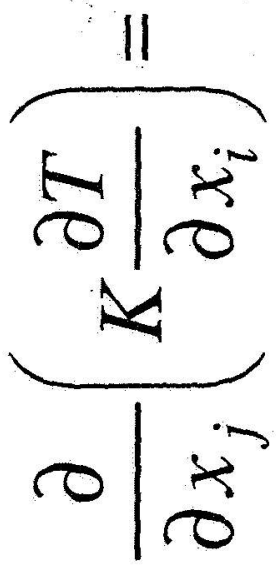

1
$E$
$\because E$

$\frac{1}{2}$

ह

(1)

$\infty$

$\ddot{\sigma}$

N

$\frac{0}{0}$
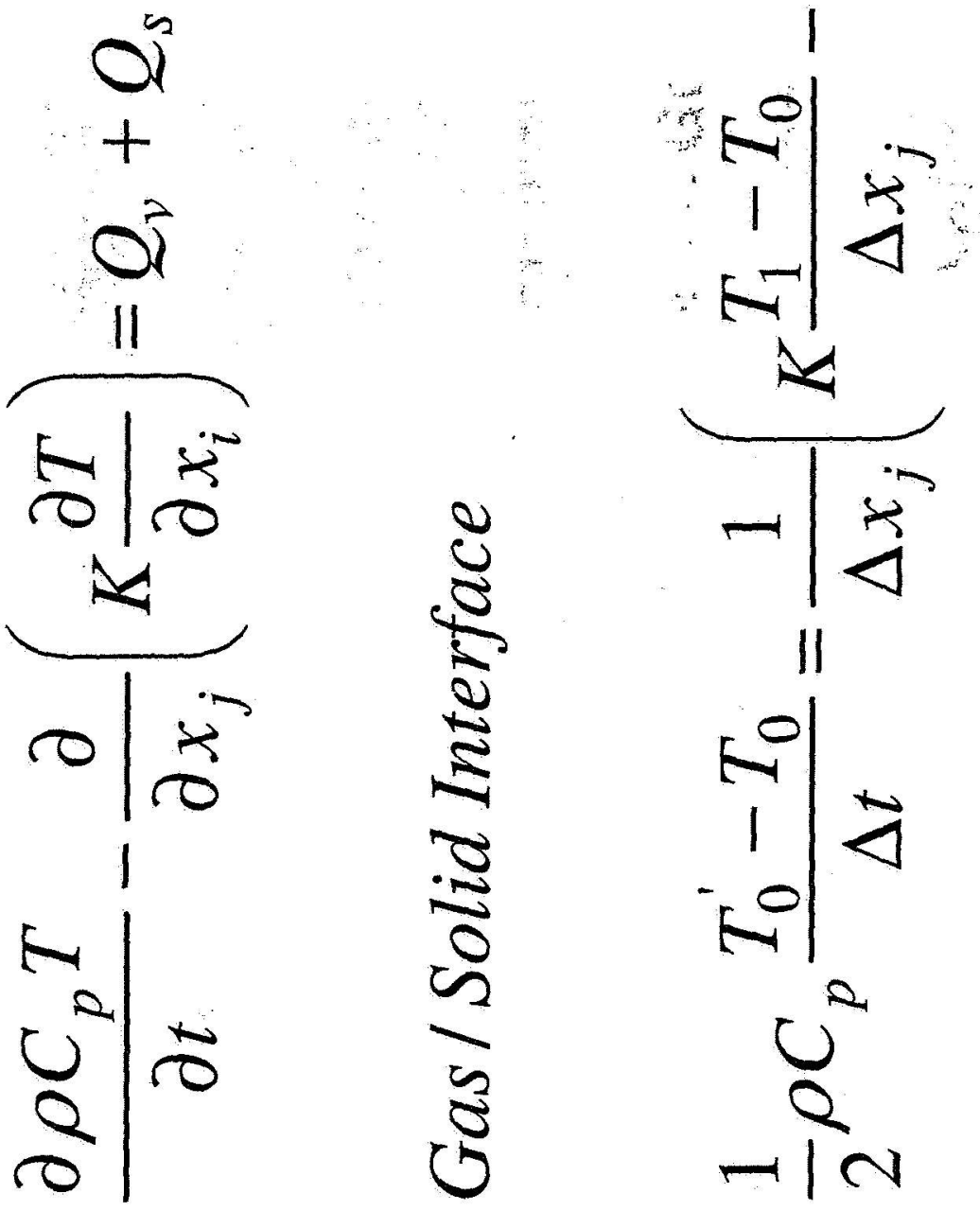

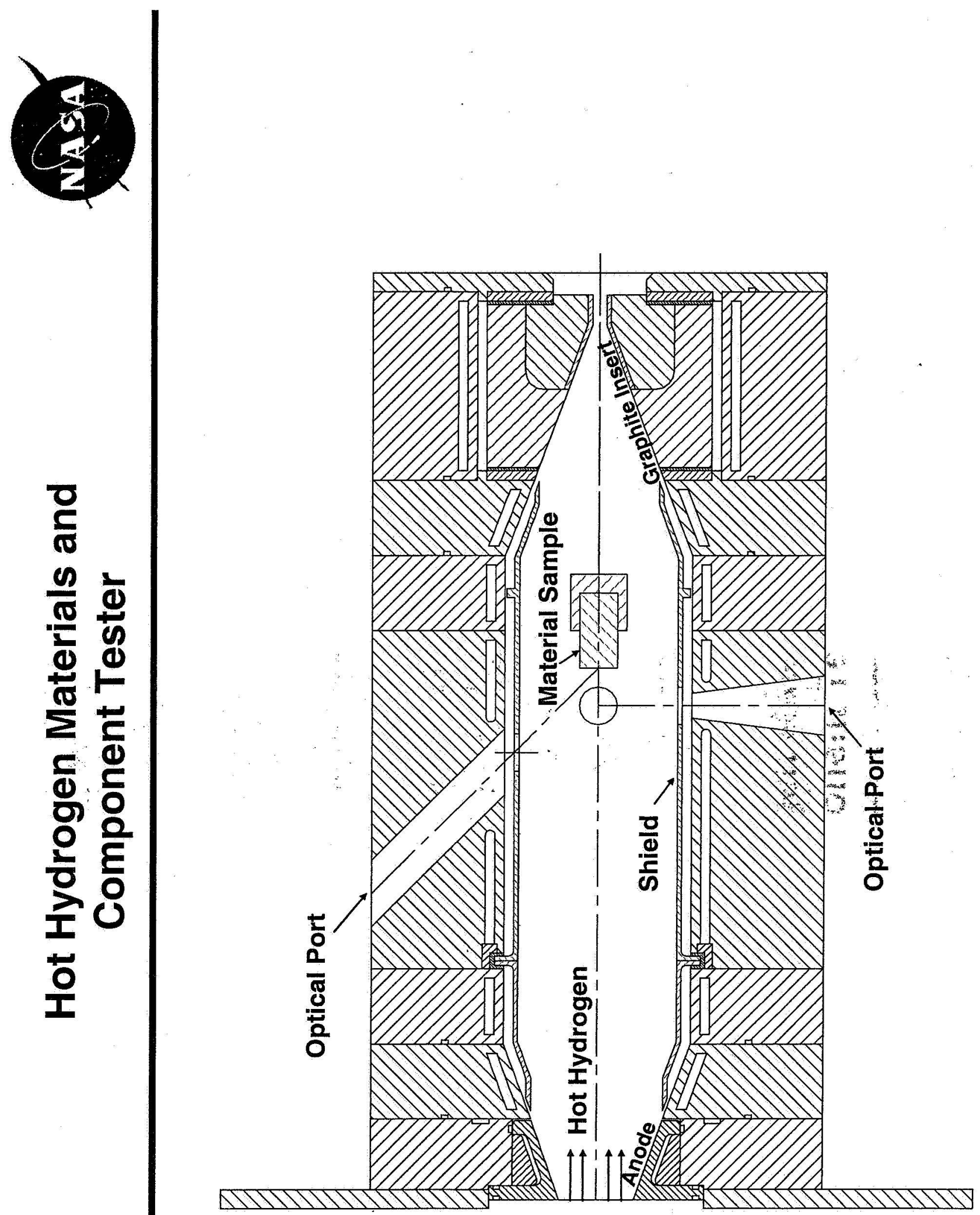


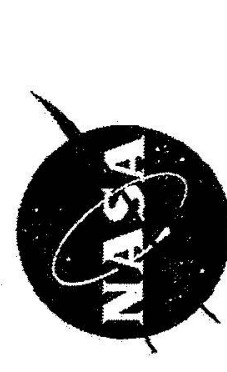

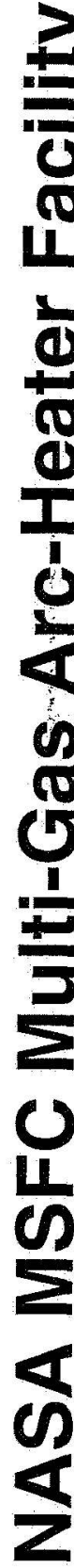

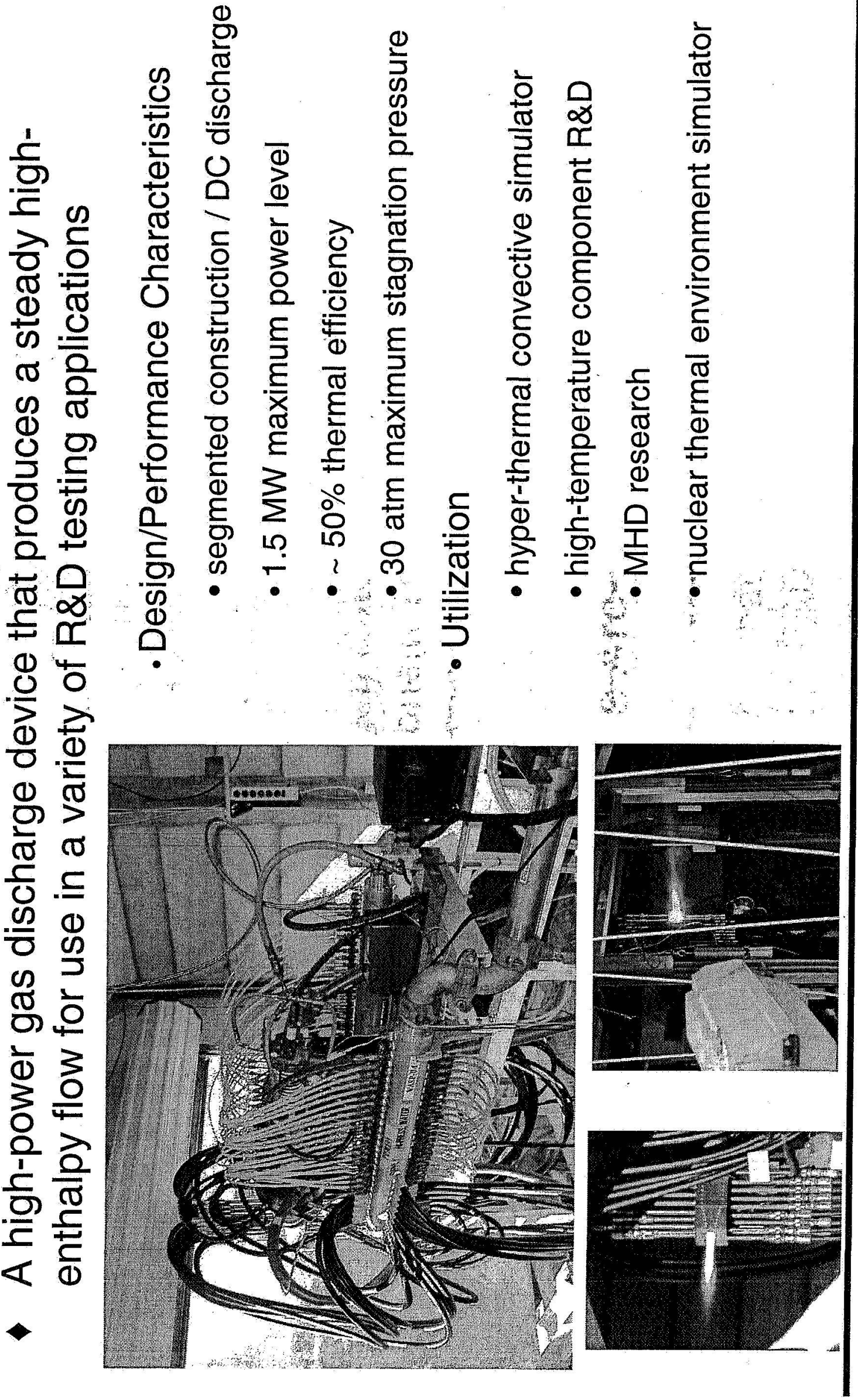




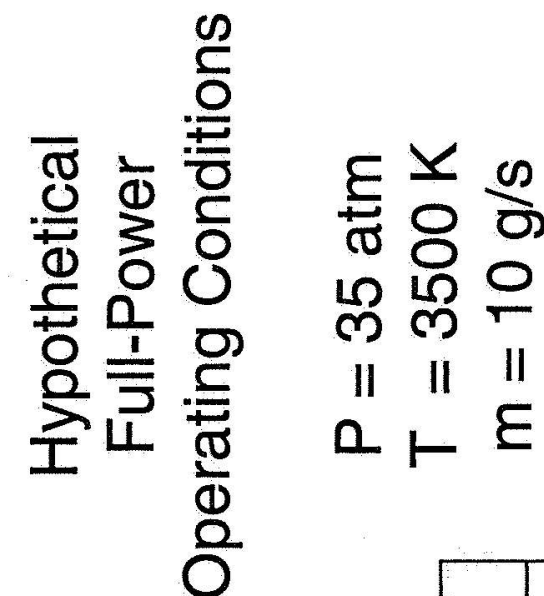

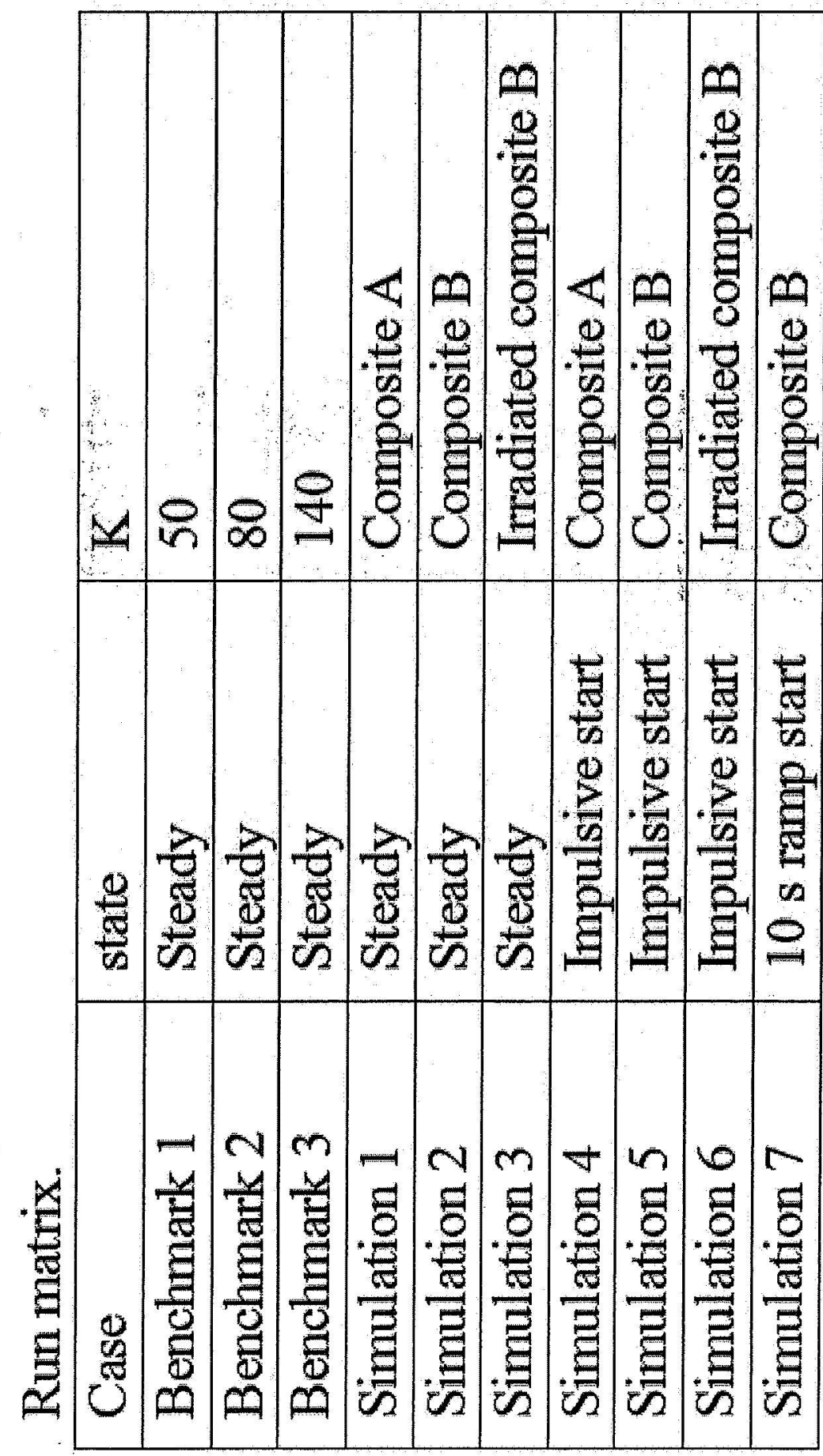




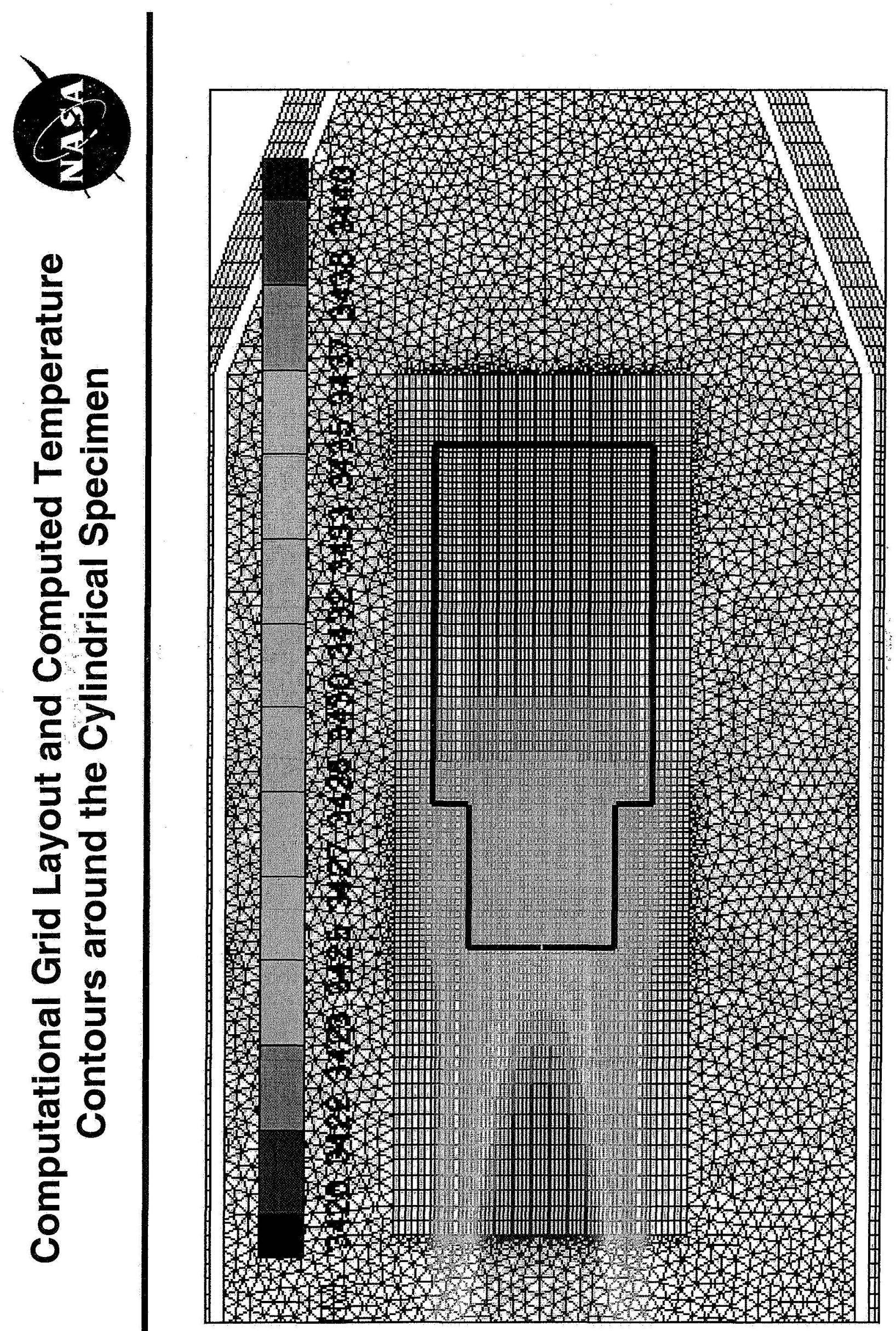



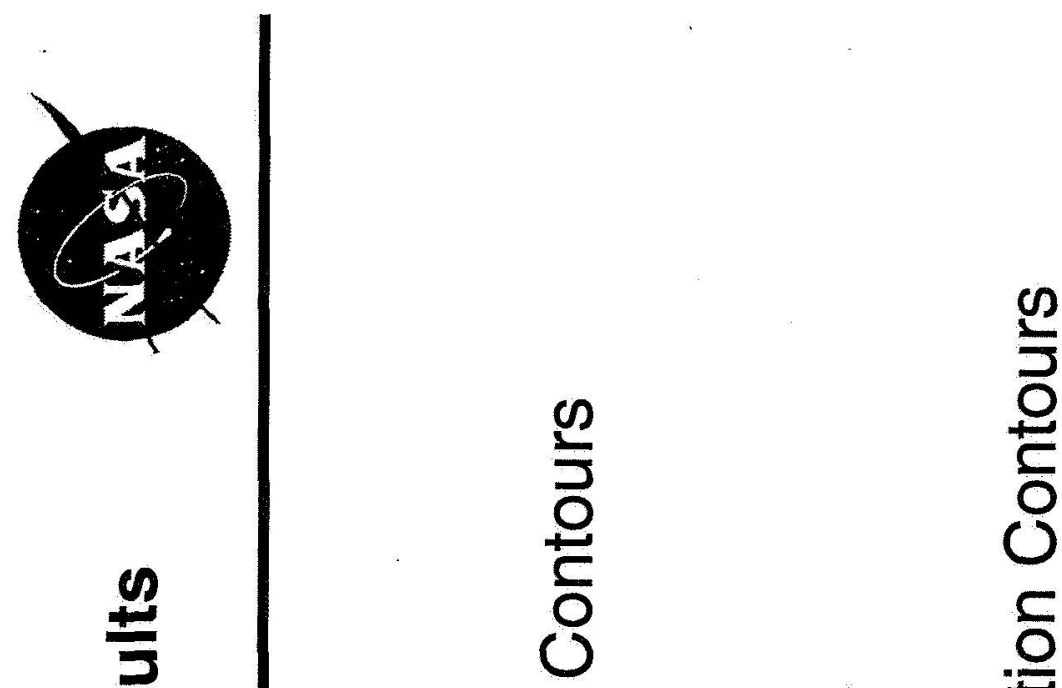

$\frac{0}{5}$

$\frac{5}{0}$

a

$\pm$

แับ

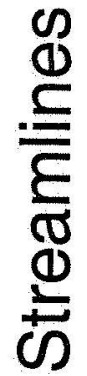

(1)

들

(1)

C)

3

$\sum$

프

10

5

ह

4
4

$\geq$

$\frac{\Phi}{50}$
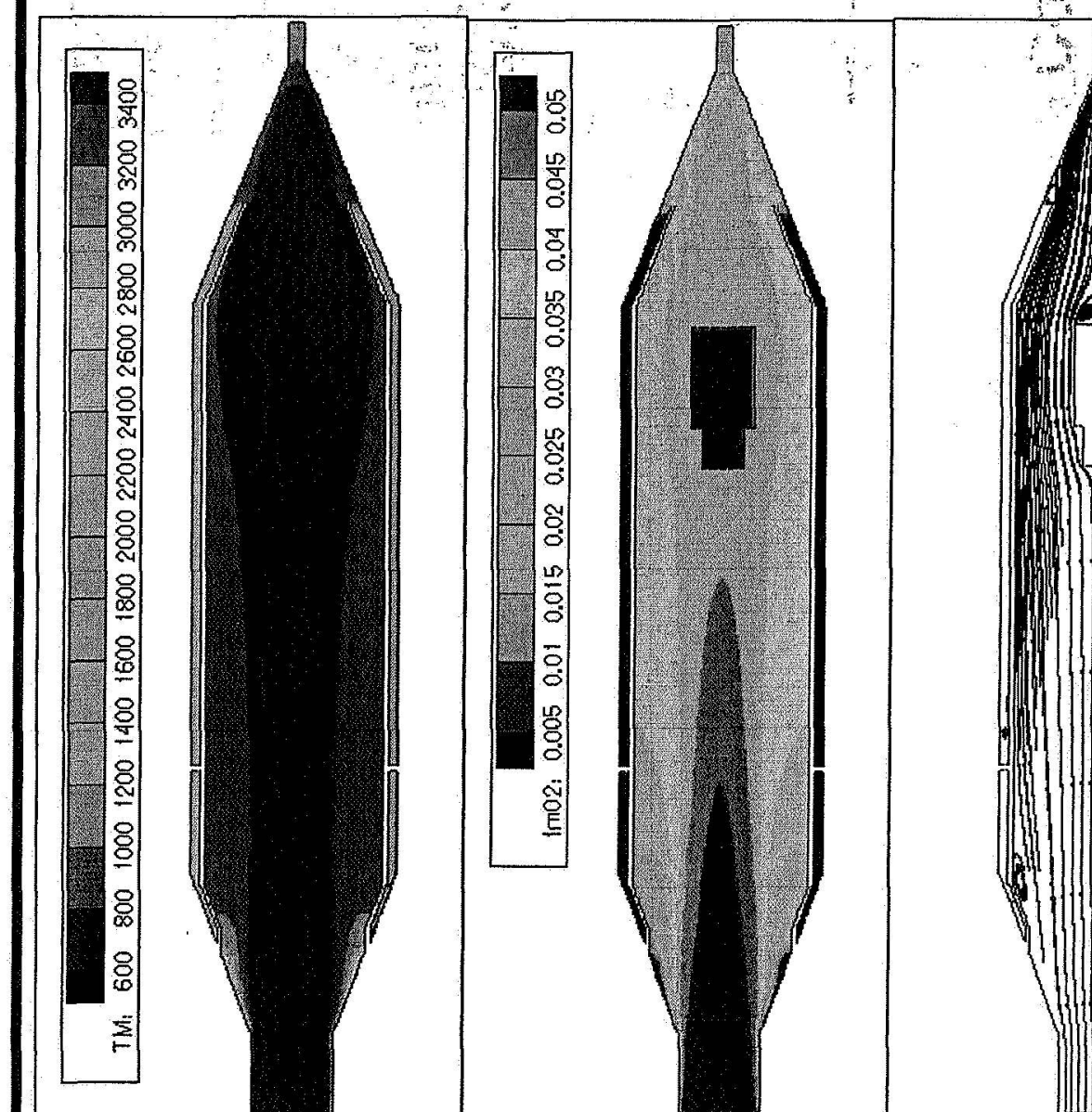


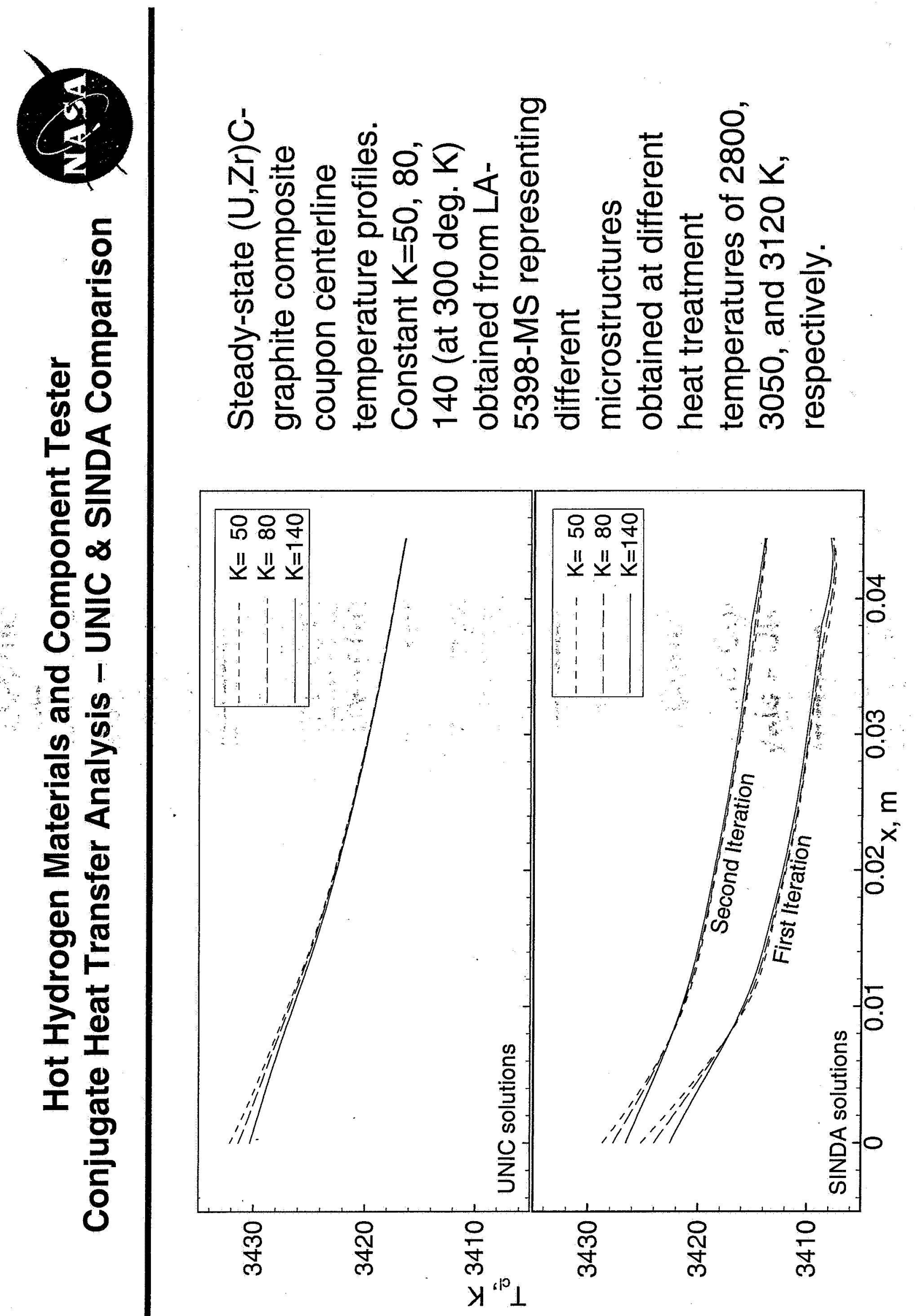



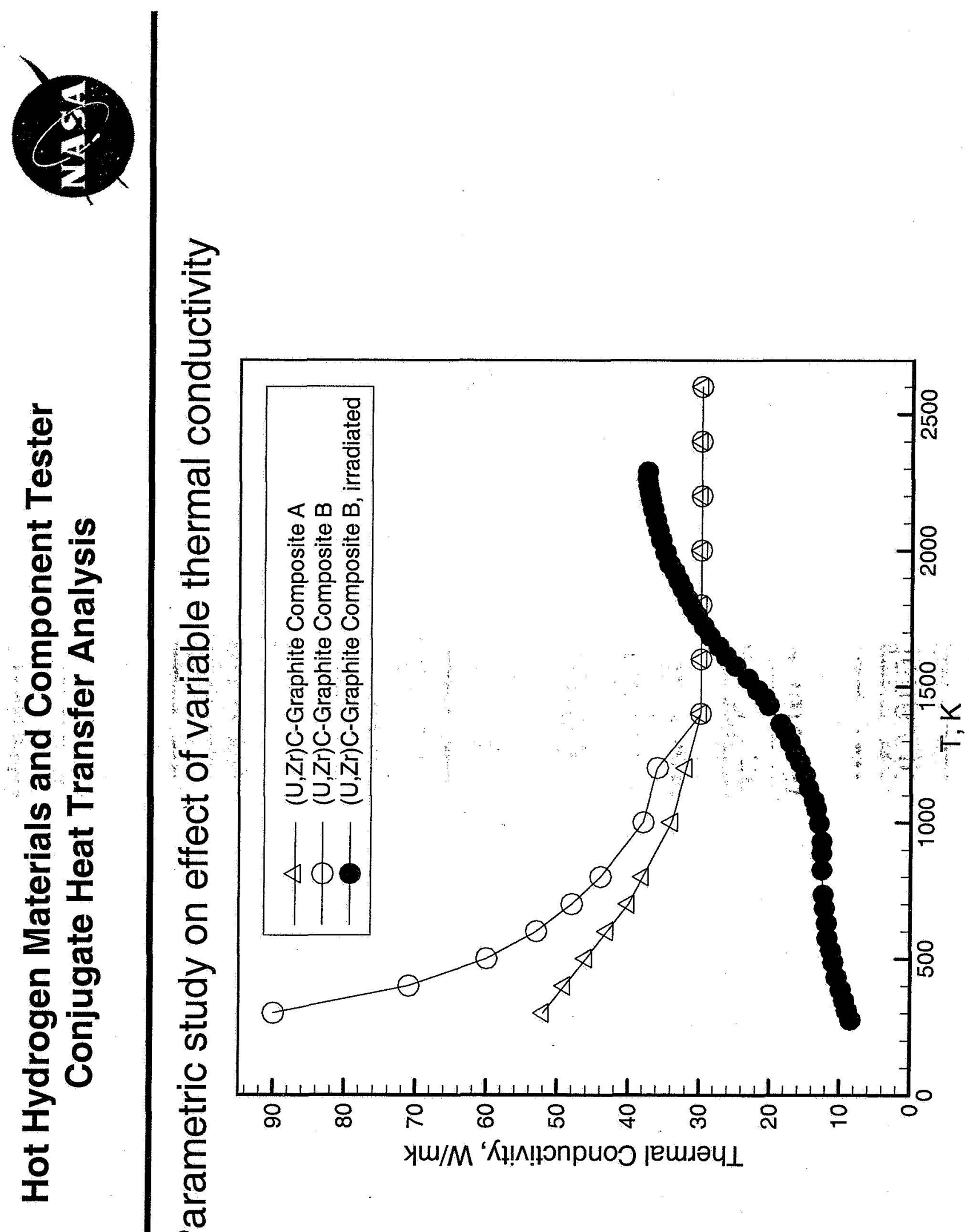

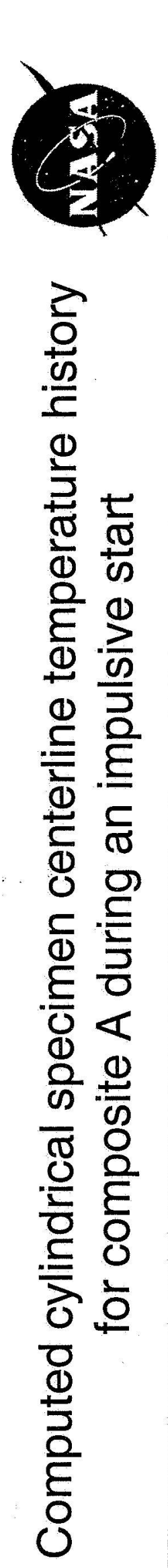

늉 O - م
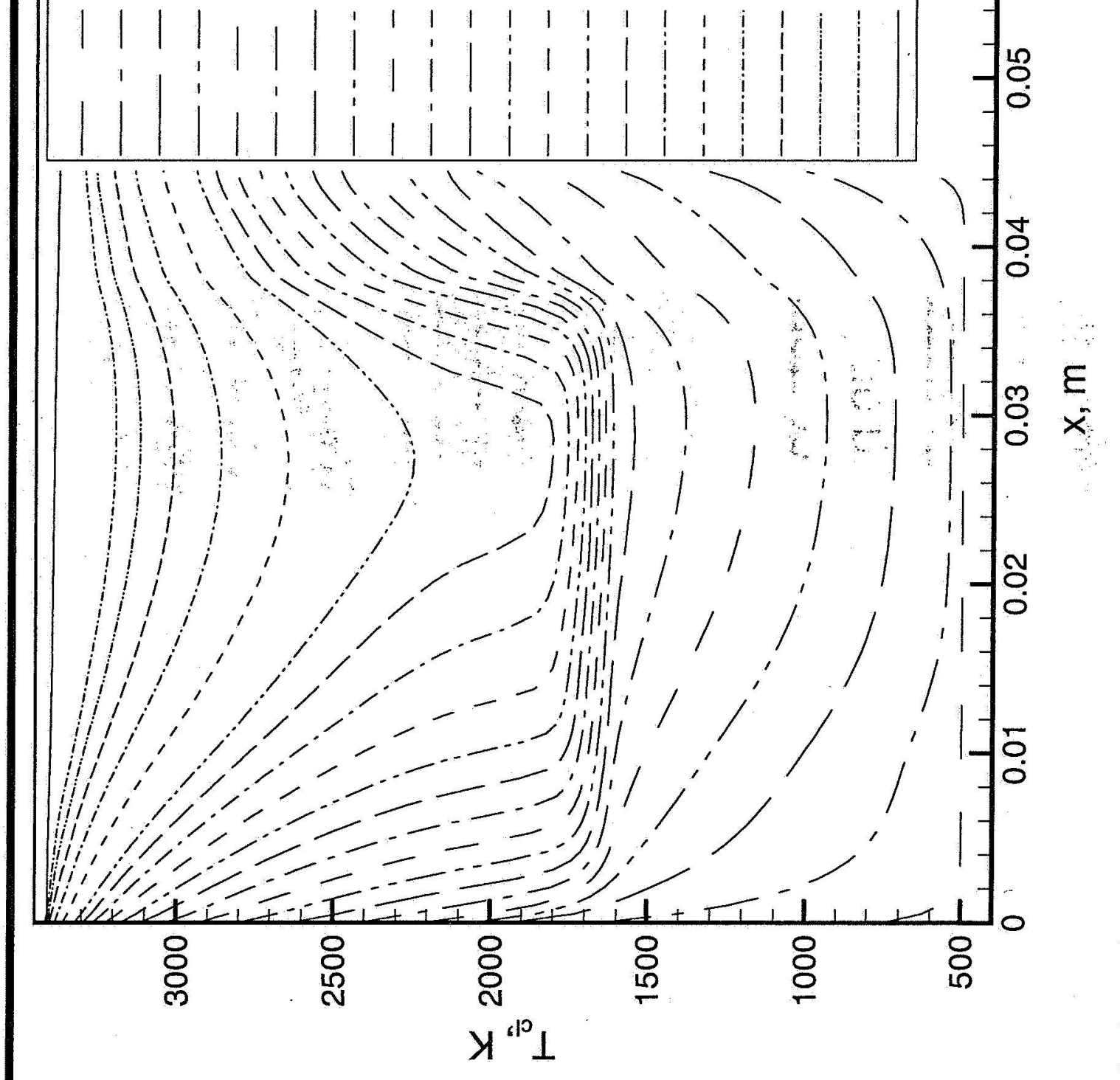

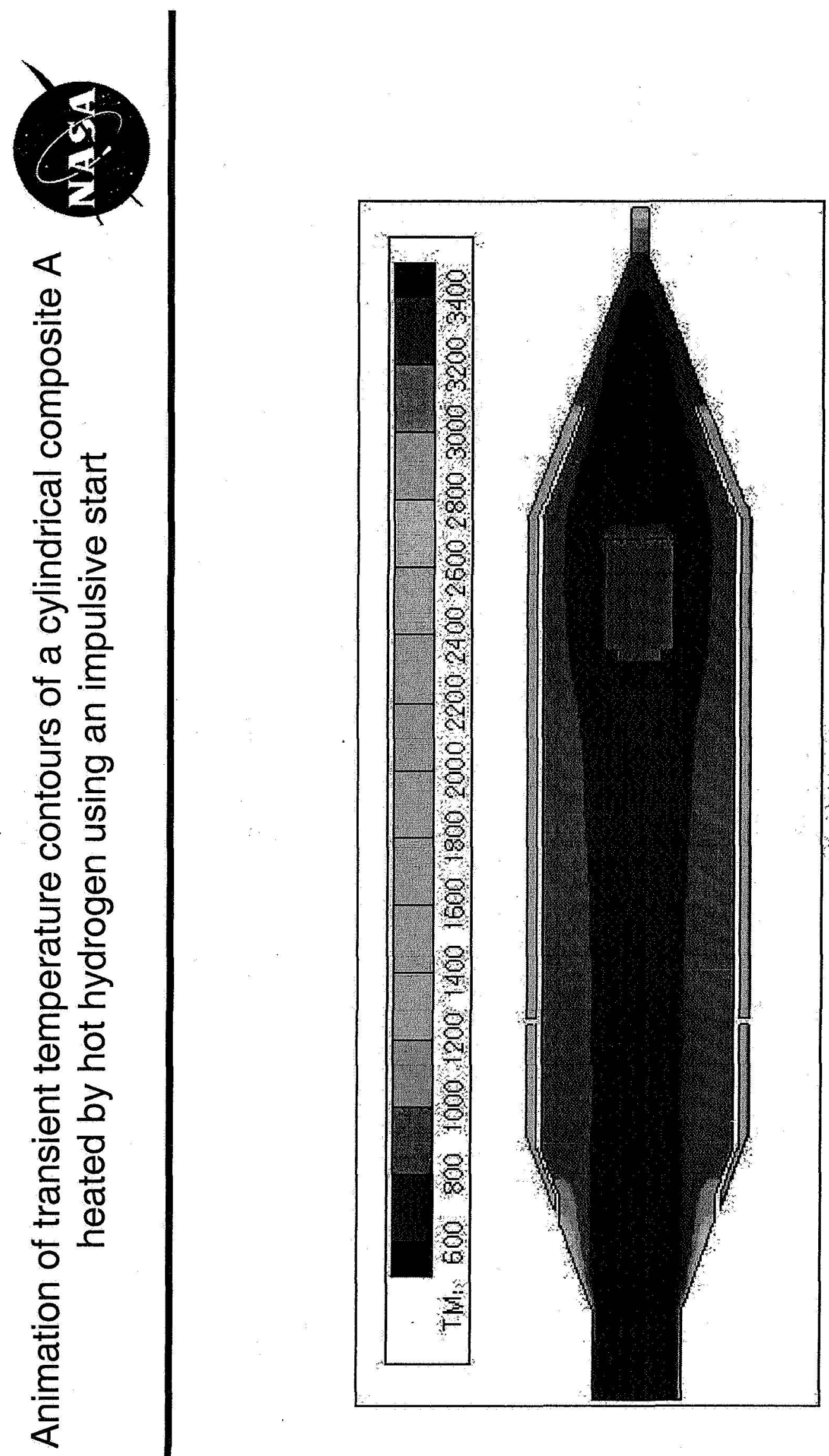


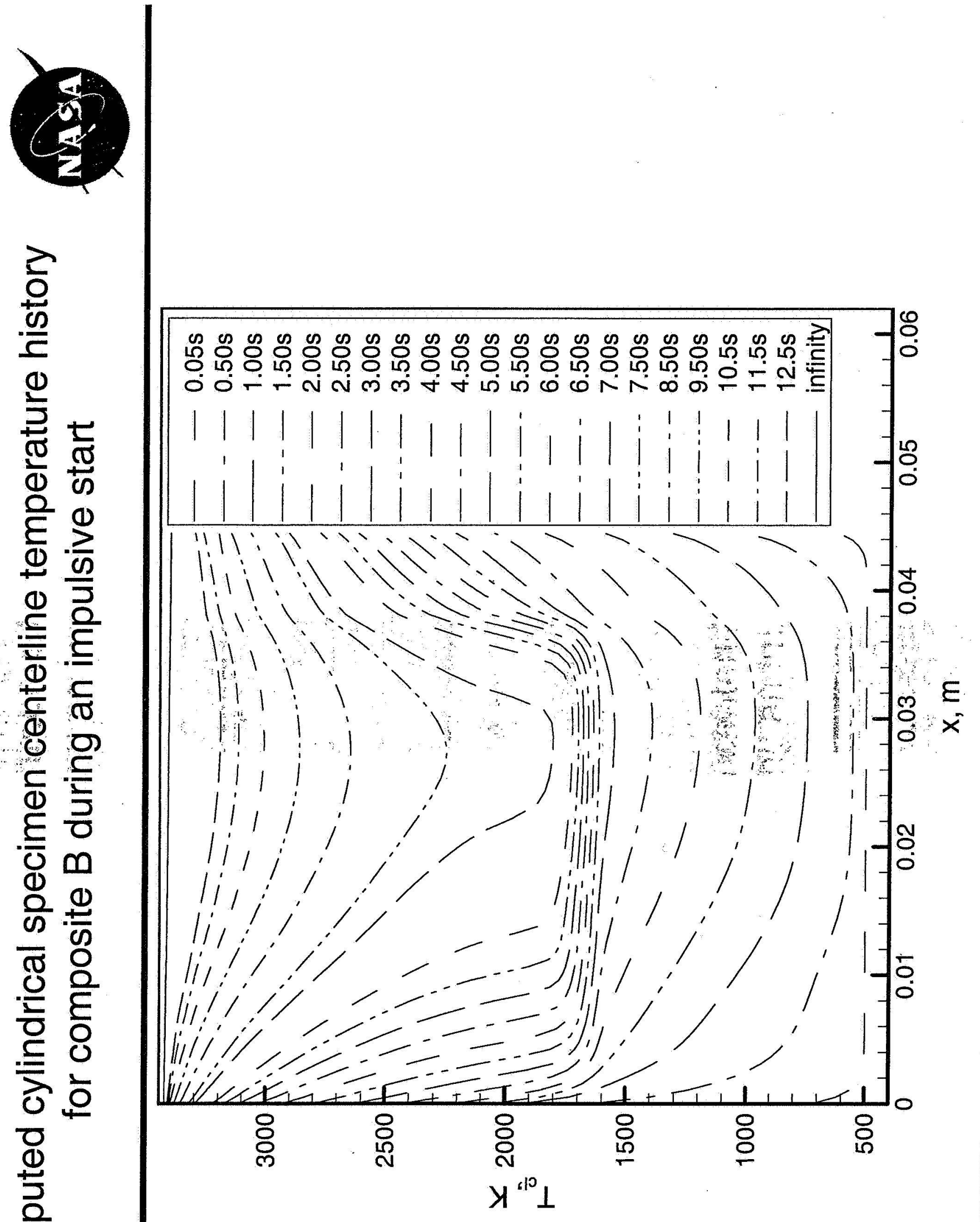



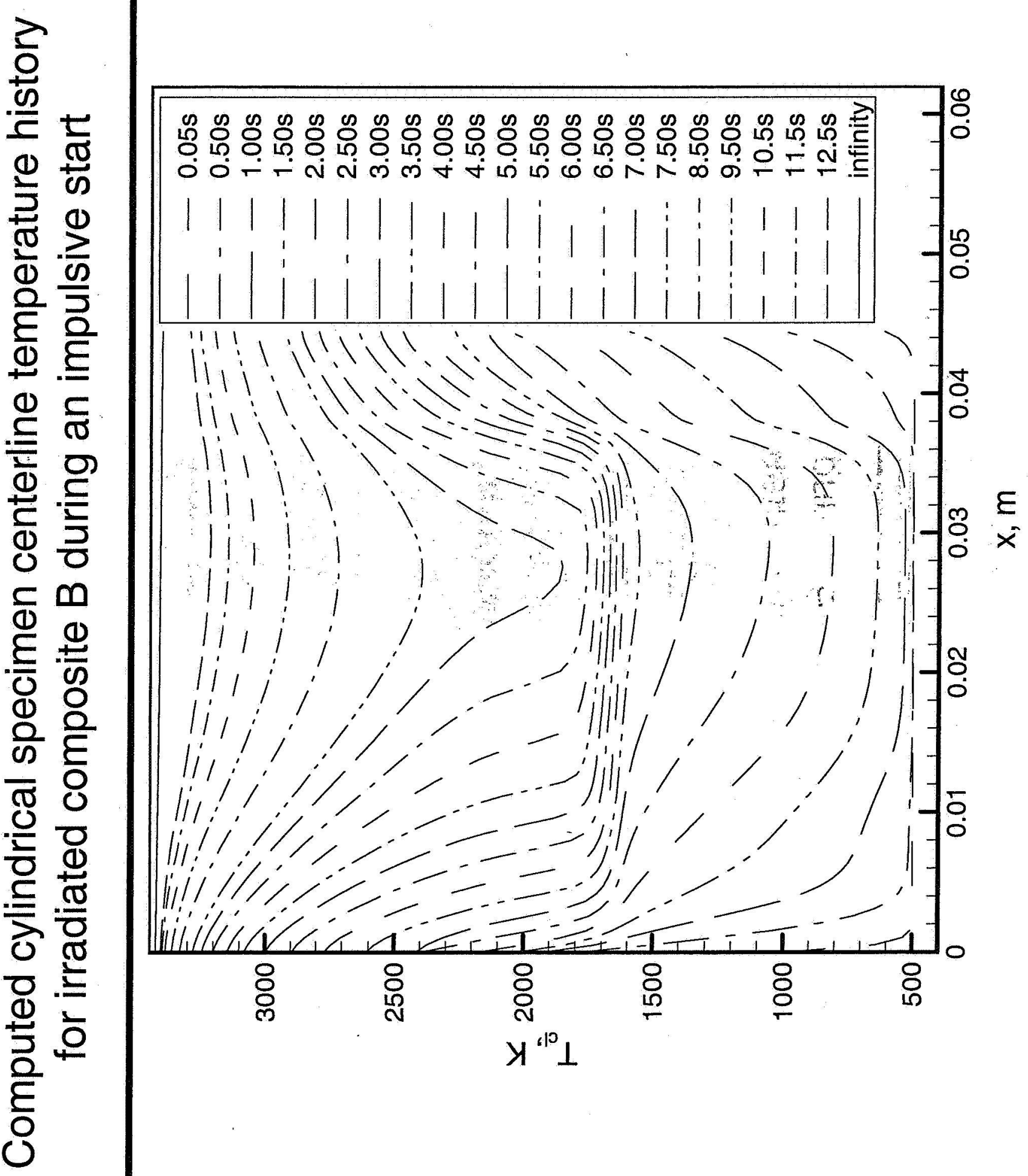


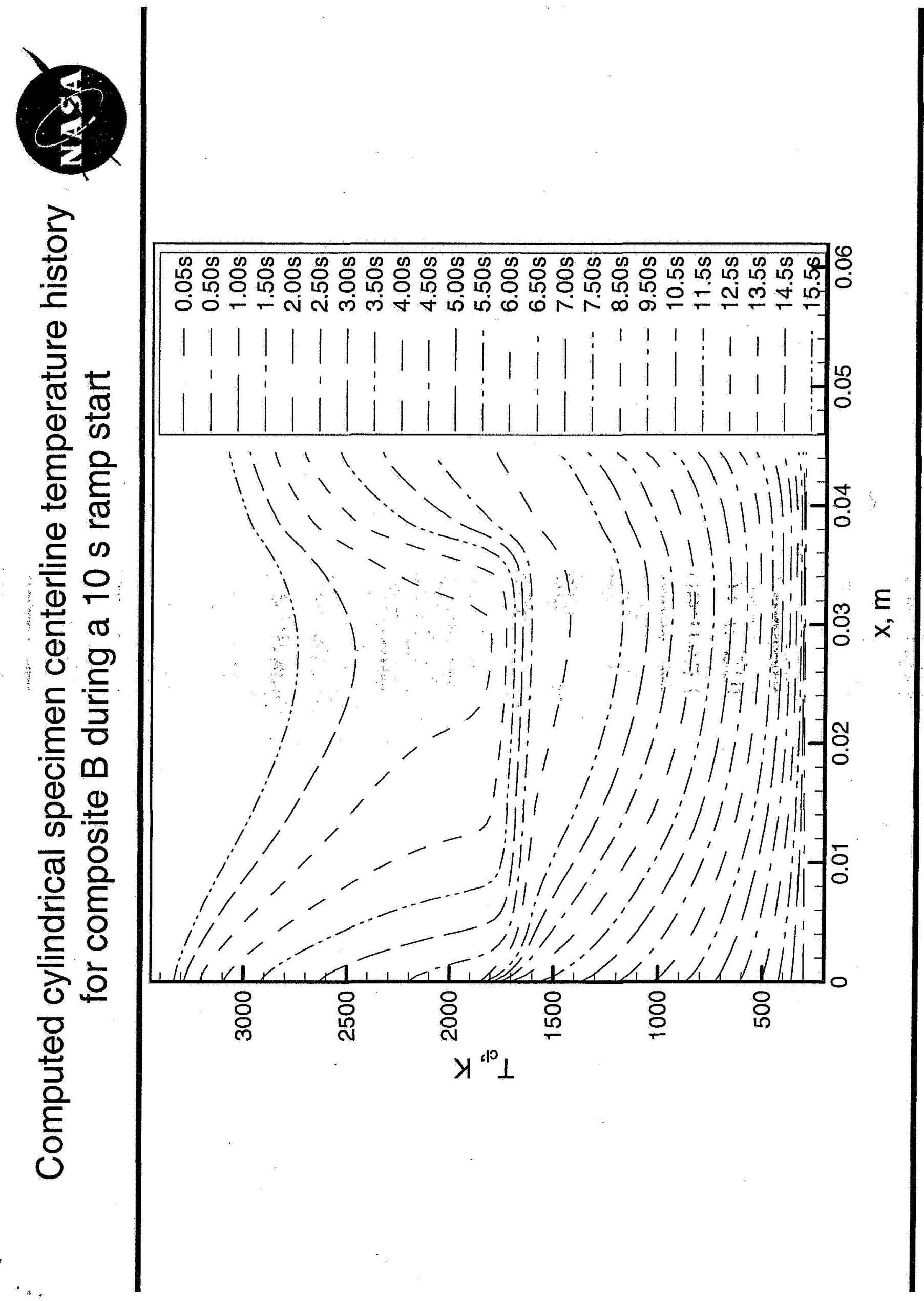




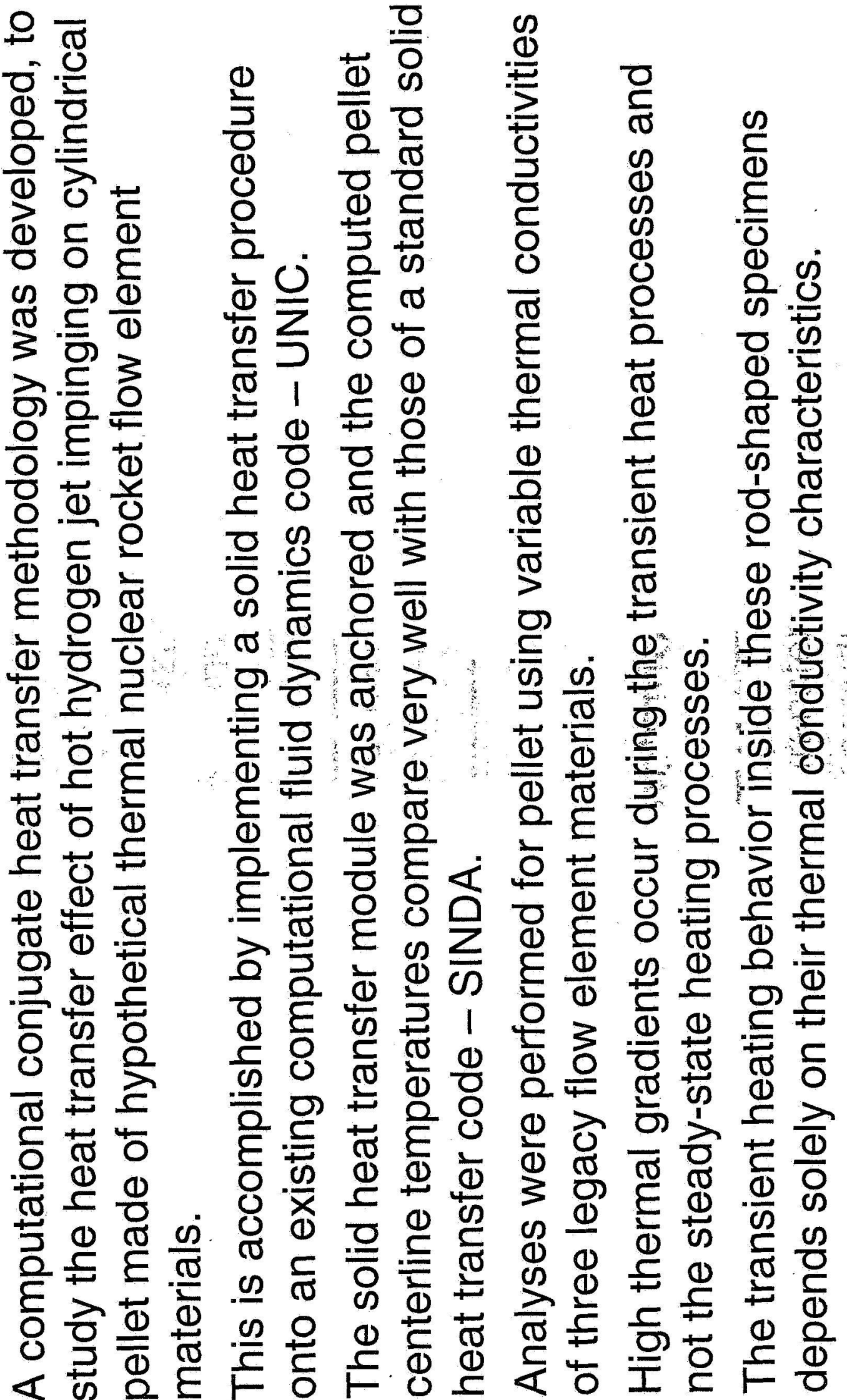

